\title{
Semiclassical analysis and passive imaging
}

\author{
Yves Colin de Verdière *
}

October 31, 2018

\begin{abstract}
The propagation of elastic waves inside the Earth provides us with informations about the geological structure of the Earth's interior. Since the beginning of seismology, people have been using waves created by earthquakes or by artificial explosions. They record the waves as functions of time using seismometers located at different stations on the Earth's surface. Even without any earthquake or explosion, a weak signal is still recorded which has no evident structure: it is a "noise". How to use these noises? This is the goal of the method of "passive imaging". The main observation is the following one: the time correlation of the noisy fields, computed from the fields recorded at the points $A$ and $B$, is "close" to the Green's function $G(\tau, A, B)$ of the wave propagation. The aim of this paper is to provide a mathematical context for this approach and to show, in particular, how the methods of semiclassical analysis can be be used in order to find the asymptotic behaviour of the correlations.
\end{abstract}

\section{Introduction}

Seismologists want to recover the physical parameters of the Earth's interior from records (called seismograms), at the Earth's surface, of the elastic waves propagating in its interior. From the mathematical point of view, it is an example of a so-called "inverse problem": the most famous inverse problems are the Calderón problem (recovering the conductance of a domain from boundary measurements) and the Kac problem (recovering a Euclidean domain from the spectrum of its Dirichlet Laplacian). The use of elastic waves created by an earthquake (resp. an artificial explosion) has been quite successful in order to recover the large scale structure (resp. the small scale structure, e.g. for oil detection). The geological structure of the Earth crust up to depths of 30-50 kilometres is much more difficult to know.

On the other hand, only a small part of the seismograms was used: this part corresponds to the propagation of well identified body and surface waves: typically the P-waves, the S-waves and the Rayleigh waves. The last part (called the "coda") of the seismograms were not used. This was a long standing question asked by K. Aki, one of the founders of modern seismology. Quite recently, it was observed by M. Campillo and A. Paul [5] that the time-correlation of coda waves is closely related to the Green's function.

Later, M. Campillo and his collaborators showed that the correlations of ambient noise already yield the Green's function [8, 24, 25]. Similar facts had been known before in acoustics [18, 31, 32, 9] and helio-seismology [12. Several theoretical results were derived in [2, 21, 22]. Applications to clock synchronisation [22] and to volcanic eruptions forecasting [4 have been developed. The paper [16] is a recent

\footnotetext{
*Institut Fourier, Unité mixte de recherche CNRS-UJF 5582, BP 74, 38402-Saint Martin d'Hères Cedex (France); http://www-fourier.ujf-grenoble.fr/ ycolver/
} 
survey. So, let us assume that we can recover the Green's function: how does it help to solve our inverse problem? Here enters semiclassics (or ray theory): the semiclassical behaviour of the Green's function contains the ray dynamics. Even more, the full Green's function is not really needed for that, its phase is sufficient. So it is natural to study the problem of passive imaging using the tools of semiclassical analysis.

The goal of my paper is to present some mathematical models for this method of passive imaging. They will be applicable to any kind of wave, but I have in mind seismic waves. Semiclassical analysis will be used as a way to get a geometric approximation to the wave propagation AND in the modelling of the source noise. I do not at all pretend that the assumptions of my models are realistic from the physical point of view. I just hope that they will help seismologists by providing another point of view and other words. On the other hand, these models put light onto several interesting mathematical problems: mode conversion, inverse spectral problems, mode-ling of random fields ...

Things work as follows: let us assume that we have a medium $X$ (a smooth manifold) and a smooth, deterministic (no randomness in it) linear wave equation in $X$. We hope to recover (part of) the geometry of $X$ from the wave propagation on it. We assume that there is somewhere in $X$ a source of noise $\mathbf{f}(x, t)$ which is a stationary random field. This source generates, by the (linear) wave propagation, a field $\mathbf{u}(x, t)$ (assumed to be scalar in this introduction). This field $\mathbf{u}$ is recorded at different points $A, B, \cdots$ on long time intervals. We want to get some information on the propagation of waves from $B$ to $A$ in $X$ from the correlation

$$
C_{A, B}(\tau)=\lim _{T \rightarrow+\infty} \frac{1}{T} \int_{0}^{T} \mathbf{u}(A, t) \overline{\mathbf{u}}(B, t-\tau) d t
$$

which can be computed numerically from the fields recorded at $A$ and $B$. In seismology the medium $X$ will be the Earth, the waves are elastic waves, the main source noise is due to the interaction between the ocean or the atmosphere with the Earth's crust, and the field is recorded at the Earth's surface.

It turns out that $C_{A, B}(\tau)$ is closely related to the deterministic Green's function of the wave equation in $X$. It means that one can hope to recover, using Fourier analysis, the propagation speeds of waves between $A$ and $B$ as a function of the frequency, and, more precisely, the Hamiltonian or, more generally, the so-called dispersion relation.

If the wave dynamics is time reversal symmetric, the correlation admits also a symmetry by changing $\tau$ into $-\tau$; this observation has been used for clock synchronisation, see [22].

The goal of this paper is to give precise formulae for $C_{A, B}(\tau)$ in the high frequency limit, under some assumptions on the source $\mathbf{f}$. We will assume that the source field is a stationary ergodic random field, the covariance of which $K(x, y, s-$ $\left.s^{\prime}\right)=\mathbb{E}\left(f(x, s) \bar{f}\left(y, s^{\prime}\right)\right)$ is given by the Schwartz kernel of a pseudodifferential operator. This is a strong assumption, which implies in particular a rapid decay of the correlations of the source $\mathbf{f}$ away from the diagonal.

More precisely, we have two small parameters, one of them entering into the decorrelation distance of the source noise, the other one in the high frequency propagation (the ray method). The fact that both are of the same order of magnitude is crucial for the method. As a result, the method works well in certain frequency ranges of the wave propagation. From the physical point of view, the main result is that, with some assumptions on the support of the source noise, one can recover the dispersion relation (the ray dynamics) in some frequency interval. A mathematical statement will be that, for $\tau>0, C_{A, B}(\tau)$ is close to the Schwartz kernel of $\Omega(\tau) \circ \Pi$ where $\Pi$ is a suitable pseudodifferential operator ( $\Psi$ DOfor short), the 
principal symbol of which can be explicitly computed, and $\Omega(\tau)$ is the (semi)group of the (damped) wave propagation. This closeness is in general only meant in the $L^{2}$ sense, but can be pointwise if the damping is strong enough. It implies that we can recover the dispersion relation, i.e. the classical dynamics, from the knowledge of all two-points correlations.

The waves are recorded at the boundary, it implies that the main part of the recorded noisy fields is coming from surface waves. So we end up with another inverse problem: how does the dispersion relation of the elastic surface waves determine the structure of the Earth's interior? We will discuss the effective (dispersive) ray dynamics of guided surface waves. The corresponding ideal inverse spectral problem has been solved in [7] from which we recall the main result.

I guess that our results can be interesting because they provide a quite general situation in which the source is not a white noise and for which we can still recover the most interesting part of the Green's function (the phase).

Let us also mention on the technical side that, rather than using mode decomposition, we prefer to work directly with the dynamics; in other words, we need a time dependent rather than a stationary approach. Mode decomposition is often useful, but it is of no much help for a general system with no particular symmetry.

For clarity, we will first discuss the nonphysical case of a first order wave equation like the Schrödinger equation, then the case of more common wave equations (acoustic, elastic).

In order to make the paper readable by a large audience, we have tried to make it self-contained by including sections on pseudodifferential operators and on random fields.

\section{Contents}

- In Section1, we start with a quite general setting and discuss a general formula for the correlation (Equation (20). This formula will be made more precise in several cases in the following Sections.

- The goal of Section 2 is to introduce the basic examples which we will discuss in the paper.

- In Section 3, we introduce a large family of anisotropic random fields and show the relation between their power spectra and their Wigner measures. They are build from white noises using the Radonification process: our random fields will be the images of some white noises by some suitable linear operators.

- Section 4 is devoted to the case of a scalar field driven by a first order in time differential equation. This case is quite academic, but will be used later for standard wave equations. We will discuss first the (nonrealistic) case where the source is a white noise, then the semiclassical case assuming the absence of mode conversion.

- In Section 5 we discuss the case of multicomponent wave equations: we will discuss first the (nonrealistic) case where the source is a white noise, then the semiclassical case using a reduction to the case of scalar fields.

- In Section 6, going back to the beginning of the story, we discuss the question of the correlations of codas in the framework of "quantum chaos".

- In Section 7 we focus on the case of seismology and discuss the remarkable fact that the correlation of the surface waves is enough to image the Earth's crust, using a waveguide model of the crust. 
- Finally, there are four appendices:

- Appendix A on pseudodifferential operators,

- Appendix B on classical dynamics, dispersion relations and generating functions,

- Appendix C on Egorov Theorem for long times,

- Appendix D on time averages versus ensemble averages, i.e. ergodicity of the source field.

As can been seen in the paper,several interesting mathematical problems come out of our discussions; let us mention

- Mode conversions associated with an eigenvalue going into the continuous spectrum (Section 7.3).

- Extension of the large time Egorov Theorem, with Fourier integral operators replacing $\Psi D O$ 's, in order to be able to properly discuss the case of a source noise localized on a submanifold.

- The large time behaviour of the solution of a wave equation with initially localised Cauchy data, like in an earthquake (Section 6).

Acknowledgement: I would like to thank Michel Campillo and his colleagues from LGIT for discussions and collaborations. I also thank the referee for his great help in order to improve the initial manuscript.

\section{A general formula for the correlation}

In this quite mathematical Section, we present the general context and a general formula for the correlation which makes quite explicit the relation with the Green's function: we will do that on the level of operators and on the level of more concrete functions, the integral (or Schwartz) kernels of the operators. Our model is quite general: it includes linear wave equations with some attenuation and a source noise on the right hand side of the equation.

We will consider the following general damped wave equation:

$$
\frac{d \mathbf{u}}{d t}+\frac{i}{\varepsilon} \hat{H} \mathbf{u}=\mathbf{f}
$$

\subsection{Operators level}

We make the following assumptions:

1. The parameter $\varepsilon>0$ can be either fixed (say $\varepsilon=1$ ) or can be a semiclassical parameter going to $0^{+}$, like $\hbar$ in the Schrödinger-like case, or the inverse of the frequency for the wave equation.

2. The operator $i \hat{H} / \varepsilon$ is the infinitesimal generator of a strongly continuous group (see [36], Chap. IX)

$$
\Omega(t)=" \exp (-i t \hat{H} / \varepsilon) "
$$

acting on some Hilbert space $\mathcal{H}$.

3. $\Omega(t)$ is a contraction group: there exists $T_{\text {att }}>0$, called the attenuation time, and $C>0$ so that the operator norm of $\Omega(t)$ satisfies

$$
\forall t \geq 0,\|\Omega(t)\| \leq C e^{-t / T_{\text {att }}}
$$


4. The source noise $\mathbf{f}$ is a stationary (see Section 3.5) random field $\mathbf{f}: \mathbb{R} \rightarrow \mathcal{H}$. We assume that $\mathbb{E}\left(\|\mathbf{f}(0)\|^{2}\right)<\infty(1)$ and the covariance of $\mathbf{f}$ is given by (2)

$$
\mathbb{E}\left(\left|\mathbf{f}(s)><\mathbf{f}\left(s^{\prime}\right)\right|\right)=K\left(s-s^{\prime}\right)
$$

with $K(t) \in \mathcal{L}(\mathcal{H})(3)$, a continuous function of $t$ with compact support $\{|t| \leq$ $\left.t_{0}\right\}$ (4).

Definition 1.1 The causal solution of Equation (1) is the solution given by $u=$ $\mathbf{G} f$, with $\mathbf{G}$ a linear operator, satisfying $u(., t)=0$ if $f(., s)$ vanishes for $s \leq t$.

For physical reasons, it is appropriate to take for $\mathbf{u}$ the causal solution of Equation (11). The field $\mathbf{u}$ is then stationary, and given by

$$
\mathbf{u}(t)=\int_{0}^{\infty} \Omega(s) \mathbf{f}(t-s) d s .
$$

Using Assumptions 3. and 4, one can show that $\mathbf{u}(t)$ is almost surely defined, and its covariance, defined by

$$
C(\tau)=\mathbb{E}(|\mathbf{u}(0)><\mathbf{u}(-\tau)|),
$$

is a continuous linear operator on $\mathcal{H}$.

Theorem 1.1 The covariance $C(\tau)$ is given by

- for $\tau>t_{0}$,

$$
C(\tau)=\Omega(\tau) \Pi \quad \text { with } \quad \Pi=\int_{0}^{\infty} \Omega(s) \mathcal{L} \Omega^{\star}(s) d s \quad \text { and } \quad \mathcal{L}=\int_{-t_{0}}^{t_{0}} \Omega(-u) K(u) d u
$$

- for $\tau<0$,

$$
C(\tau)=(C(-\tau))^{\star}
$$

Assuming $\mathbf{f}$ to be ergodic (see Section 3.5), the covariance $C(\tau)$ is also given almost surely by the time average:

$$
C(\tau)=\lim _{T \rightarrow \infty} \frac{1}{T} \int_{0}^{T}|\mathbf{u}(t)><\mathbf{u}(t-\tau)| d t .
$$

In order to perform effective passive imaging, people use the previous formula for a large enough $T$ and ... computers.

\subsection{Kernels level}

In order to have concrete functions and not only distributions, we will make some smoothness assumptions:

- $X$ is a smooth compact manifold of dimension $d$ with a smooth measure $|d x|$. For applications to seismology $X$ will be the Earth or (in Section77) the Earth's surface.

\footnotetext{
${ }^{1} \mathbb{E}$ denotes the ensemble average or expectation value

${ }^{2}$ We use Dirac's bra-ket notation, $\mid f>$ is a vector, $<f \mid$ is the Hermitian scalar product with $f$, so that $|f><g|$ is the rank one linear operator: $x \rightarrow<g \mid x>f$

${ }^{3} \mathcal{L}(E, F)$ is the space of continuous linear maps from the Banach space $E$ into the Banach space $F$ with the norm topology and $\mathcal{L}(E):=\mathcal{L}(E, E)$

${ }^{4}$ The assumption on compact support could be replaced by some suitable decay in $t$. It means that the source noise is uncorrelated for large enough times
} 
- $\mathbf{u}(x, t), x \in X, t \in \mathbb{R}$ is the field (scalar or vector valued) with values in $\mathbb{C}^{N}$ $\left(\right.$ or $\left.\mathbb{R}^{N}\right)$.

- The generator of the group $\Omega(t)$ is an elliptic (see Definition 10.1) differential (or pseudodifferential) linear operator $\hat{H}$ acting with a dense domain of a Hilbert space $\mathcal{H} \subset \mathcal{D}^{\prime}\left(X, \mathbb{C}^{N}\right)$.

- The source $\mathbf{f}$ is a smooth stationary random field on $X \times \mathbb{R}$ with values in $\mathbb{C}^{N}\left(\right.$ or $\left.\mathbb{R}^{N}\right)$. Its matrix valued correlation kernel is the smooth function on $X \times X \times \mathbb{R}$, with values in $\operatorname{End}\left(\mathbb{C}^{N}\right)$, given by

$$
\mathbb{E}\left(\mathbf{f}(x, s) \otimes \mathbf{f}^{\star}\left(y, s^{\prime}\right)\right)=K\left(s-s^{\prime}, x, y\right) .
$$

Definition 1.2 The propagator or Green's function $P$ is the Schwartz kernel of $\Omega(t)$ defined by:

$$
(\Omega(t) \mathbf{v})(x)=\int_{X} P(t, x, y) \mathbf{v}(y)|d y| .
$$

The propagator $P$ satisfies

$$
\int_{X} P(t, x, y) P(s, y, z)|d y|=P(t+s, x, z)
$$

which comes from the group relation $\Omega(t+s)=\Omega(t) \circ \Omega(s)$. The causal solution of Equation (11) is then given by

$$
\mathbf{u}(x, t)=\int_{0}^{\infty} d s \int_{X} P(s, x, y) \mathbf{f}(t-s, y)|d y| .
$$

Due to the ellipticity of $\hat{H}$, this is a smooth function.

The kernel of $C(\tau)$ is defined by:

$$
C_{A, B}(\tau)=\mathbb{E}\left(\mathbf{u}(A, 0) \otimes \mathbf{u}(B,-\tau)^{\star}\right),
$$

where $A, B \in X$ and $C_{A, B}(\tau) \in \operatorname{End}\left(\mathbb{C}^{N}\right)$.

From Equation (2), we get an explicit integral formula for $C_{A, B}(\tau)$ in terms of $P(t, x, y)$ and $K(t, x, y)$; Equation (2) can be written for $\tau>t_{0}$ as follows (5):

$$
C_{A, B}(\tau)=[\Omega(\tau) \Pi](A, B)
$$

\section{Examples}

In this Section, we will present the examples of "damped wave equations" which will be used in this paper. We start with concrete examples and go then to more general semiclassical examples in the scalar case as well as in the multi-component case.

\subsection{Damped Schrödinger equation}

Let $X$ be a smooth compact boundary-less Riemannian manifold whose LaplaceBeltrami operator is denoted by $\Delta$. Let us give $a: X \rightarrow \mathbb{R}$ a smooth non negative function, $V$ a smooth real valued function on $X, \hbar$ a non negative constant, and $\hat{K}=-\hbar^{2} \Delta+V(x)$, and take:

$$
\frac{\hbar}{i}\left(u_{t}+a(x) u\right)+\hat{K} u=g .
$$

\footnotetext{
${ }^{5}$ If $R$ is an operator we will denote by $[R](x, y)$ its Schwartz kernel
} 
It is a particular case of Equation (10) where $\varepsilon=\hbar, \hat{H}=\hat{K}+\frac{\hbar}{i} a(x), f=\frac{i}{\hbar} g$ and the Hilbert space is $L^{2}(X)$.

Let us note for future use that, if $\hbar \rightarrow 0$, the principal symbol (see Appendix A, Section 8.1) of our equation reads $\omega+\|\xi\|^{2}+V(x)$ where our (extended) phase space is $T^{\star}(X \times \mathbb{R})$ with canonical coordinates $(x, \xi, t, \omega)$ and $a(x)$ is a subprincipal term entering in the transport equation, but not in the classical dynamics (see Appendix A). The attenuation time can be taken as $T_{\text {att }}=1 / \inf _{x \in X} a(x)$. Let us note that this equation has no physical meaning, it is just a kind of toy model.

\subsection{Damped wave equations}

As in the previous Section, $X$ is a compact Riemannian manifold. Let us start with

$$
u_{t t}+a u_{t}-\Delta u=f
$$

( $a$ is a smooth function which satisfies $\inf _{x \in X} a(x)>0$ ). It corresponds to Equation (11) if we set

$$
\mathbf{u}=\left(\begin{array}{c}
\varepsilon \sqrt{-\Delta} u \\
-i \varepsilon u_{t}
\end{array}\right), \mathbf{f}:=\left(\begin{array}{c}
0 \\
-\varepsilon^{2} f
\end{array}\right)
$$

and

$$
\hat{H}=\left(\begin{array}{cc}
0 & -\varepsilon \sqrt{-\Delta} \\
-\varepsilon \sqrt{-\Delta} & -i \varepsilon a
\end{array}\right)
$$

The Hilbert space is defined by the energy norm $\left\|\left(u_{1}, u_{2}\right)\right\|^{2}=\int_{X}\left(\left|u_{1}\right|^{2}+\left|u_{2}\right|^{2}\right)|d x|$.

Remark 2.1 It follows from [19] that, if $a(x) \geq 0$ everywhere and there exists some $T_{0}>0$ so that the support of a $(x)$ crosses every geodesic arc of length $\geq T_{0}$, then $\Omega(t)$ is a contraction group.

One can replace the very simple wave equation (6) by more sophisticated multicomponent wave equations like the equation of elastic waves. The general form will be:

$$
u_{t t}+A u_{t}-\Lambda u=0,
$$

where $u(x, t) \in \mathbb{C}^{N}, \Lambda$ is an elliptic system of degree 2 and $A$ is a differential operator of degree 0 representing the attenuation.

\subsection{Pseudodifferential equations}

This example will be the subject of Section 4

This is a generalisation of the damped Schrödinger equation (Section 2.1); we now introduce a small parameter $\varepsilon$ which, in the case of the damped Schrödinger equation, is Planck's constant $\hbar$. The semiclassical regime will correspond to $\varepsilon \rightarrow 0$,

We assume that the dynamics is generated by a pseudodifferential operator (a $\Psi$ DO, see Appendix A). Our equation looks then like:

$$
\mathbf{u}_{t}+\frac{i}{\varepsilon} \hat{H}_{\varepsilon} \mathbf{u}=\mathbf{f}
$$

with

$$
\hat{H}_{\varepsilon}=\mathrm{Op}_{\varepsilon}\left(H_{0}+\varepsilon H_{1}\right) .
$$

Here $H_{0}$ is real valued and elliptic, and is the Hamiltonian of the ray dynamics, while $\Im H_{1}<0$ provides the attenuation.

In the case of the damped Schrödinger equation (Section 2.1), we have $\varepsilon=\hbar$, $H_{0}=\|\xi\|^{2}+V(x)$ and $H_{1}=-i a(x)$. 


\subsection{Pseudodifferential systems}

This example will be the subject of Section 5

The system is of the following form

$$
\mathbf{u}_{t}+\frac{i}{\varepsilon} \hat{H}_{\varepsilon} \mathbf{u}=\mathbf{f}, \quad \text { with } \quad \hat{H}_{\varepsilon}=\mathrm{Op}_{\varepsilon}\left(H_{0}+\varepsilon H_{1}\right) .
$$

Here

- $\mathbf{u}(x, t) \in \mathbb{C}^{N}$

- $H_{0}(x, \xi)$ is an Hermitian symmetric matrix. Each eigenvalue $\lambda(x, \xi)$ of $H_{0}(x, \xi)$ gives a ray dynamics associated to a polarised wave. The polarisation bundle is the associated eigenbundle $E_{\lambda}(x, \xi)$

- $H_{1}(x, \xi)$ is a complex matrix. Let us introduce the quadratic forms $q_{\lambda}(x, \xi)$ which is the restriction of $\left\langle\Im H_{1} x \mid x\right\rangle$ to $E_{\lambda}(x, \xi)$. The condition $q_{\lambda}<0$ ensures the attenuation.

This includes

- The high frequency limit of the wave equations (6): we have

$$
H_{0}=\left(\begin{array}{cc}
0 & -\|\xi\| \\
-\|\xi\| & 0
\end{array}\right), H_{1}=\left(\begin{array}{cc}
0 & 0 \\
0 & -i a
\end{array}\right) .
$$

A similar transform can be used for more general wave equations, like the elastic wave equation with $N=6$.

- An effective pseudodifferential wave equation associated with stratified media. They are usually $\Psi$ DO's with a nontrivial dispersion relation (see Section 7).

- Phase space dependent damping included in $H_{1}$ : this is usually the case for seismic waves. The attenuation can be created by the small-scale physics which is not uniform in general.

\section{Random fields and semiclassics}

Our aim in this section is to build quite general random fields with correlation distances given by a small parameter $\varepsilon$. It seems to be natural for that purpose to use $\varepsilon$-pseudodifferential operators. We will see how to compute the generalised power spectrum using Wigner measures. We will not restrict ourselves to time-dependent random fields. The subsection 3.5 is devoted to the time-dependent case and we introduce there the notions of stationarity and ergodicity.

\subsection{Covariance of Random fields}

A random field is simply a random variable with values in some functional space, like a Hilbert space $\mathcal{H}$. We will consider random fields $f=f(\bar{\omega}) \in \mathcal{H}$ where $\bar{\omega}$ belongs to $\Omega$, the sample probability space. We will assume that $f \in L^{2}(\Omega, \mathcal{H})$ and, without loss of generality, that our fields have mean $0: \mathbb{E}(f)=0$.

The covariance of $f$ is the operator $C=\mathbb{E}(|f><f|)$ on $\mathcal{H}$. This operator is Hermitian and positive. $\mathcal{H}$ will often be a space of functions, and we will consider the Schwartz kernel $[C](x, y)$ of $C$.

If $\mathcal{H}$ is finite dimensional, it admits random fields for which the covariance is the identity operator. We call them white noises. 


\subsection{Cylindrical random variables}

For this section, see [27, Part II] and [15, Chap. IV]

Definition 3.1 A cylindrical random variable $f$ on the Hilbert space $\mathcal{H}$ is given by the following data: for each continuous linear map $A: \mathcal{H} \rightarrow E$ with $\operatorname{dim} E<\infty$, let $f_{A}$ be a random variable with values in $E$. These random variables must satisfy the following compatibility condition: if $l: E \rightarrow F$ is linear, $f_{l \circ A}=l \circ f_{A}$ (at the informal level, we can write $\left.f_{A}=A \circ f\right)$.

In particular, if $g: \mathcal{H} \rightarrow \mathbb{C}$ is of the form $g=F \circ A$ with $A: \mathcal{H} \rightarrow E$ linear, $\operatorname{dim} E<\infty$ and $F \in L^{\infty}(E)$, we can define $\mathbb{E}(g):=\mathbb{E}\left(F \circ f_{A}\right)$.

Example 3.1 Let $\mathcal{H}$ be $L^{2}(\mathbb{R} / 2 \pi \mathbb{Z}), \Omega=\{-1,+1\}^{\mathbb{Z}}$, with the product measure $\Pi\left(\frac{1}{2}(\delta(-1)+\delta(1))\right)$. Then $f_{\omega}(x)=\sum_{n \in \mathbb{Z}} \omega_{n} e^{i n x}$ is a cylindrical random variable on $\mathcal{H}$. $f$ is not a random variable with values in $\mathcal{H}$, but it is a random Schwartz distribution in the Sobolev space $H^{s}(\mathbb{R} / 2 \pi \mathbb{Z})$ for $s<-\frac{1}{2}$. Indeed, for any $\omega \in \Omega$ we have $\left\|f_{\omega}\right\|_{H^{s}}^{2}=\sum_{n}\left(1+|n|^{2}\right)^{s}<\infty$.

Definition 3.2 The Fourier transform $\widehat{w}$ of a cylindrical random variable $w$ is a function on $\mathcal{H}$ defined as follows. For each $x \neq 0$, one can take $\Pi_{x}$ the orthogonal projector on the one-dimensional subspace $\mathbb{C} x$. To this projection is associated the random variable $w_{\Pi_{x}}$ taking values in $\mathbb{C} x$. The value of the Fourier transform at $x$ is given by $\hat{w}(x)=\mathbb{E}\left(\exp \left(-i\left\langle w_{\Pi_{x}} \mid x\right\rangle\right)\right)$. Informally, we can write $\hat{w}(x)=$ $\mathbb{E}(\exp (-i\langle w \mid x\rangle))$.

To any random variable $f$ with values in $\mathcal{H}$ is associated the cylindrical random variable defined by $f_{A}=A \circ f$. If the cylindrical random variable comes from a random variable $f$ with values in $\mathcal{H}$, this random variable is unique and we will say that our cylindrical random variable "is" a random variable.

We have the important

Theorem 3.1 (Sazonov [27]) If the Fourier transform $\hat{w}$ of the cylindrical random variable $w$ is continuous and $A: \mathcal{H} \rightarrow \mathcal{K}$ is Hilbert-Schmidt 6 , then $A(w)$ is a random variable on $\mathcal{K}$. The covariance of $A(w)$ is the operator $A A^{\star}$.

\subsection{White noises in Hilbert spaces}

A white noise $w$ on the Hilbert space $\mathcal{H}$ is a cylindrical random variable on $\mathcal{H}$ of mean 0 and covariance given by the identity:

$$
\forall e, f \in \mathcal{H}, \mathbb{E}(\langle e \mid w\rangle\langle w \mid f\rangle)=\langle e \mid f\rangle .
$$

Definition 3.3 The Gaussian white noise is the white noise on $\mathcal{H}$ whose Fourier transform is $\exp \left(-\|x\|^{2}\right)$.

Sazonov's Theorem 3.1 applies in particular to the Gaussian white noise.

If the Hilbert space $\mathcal{H}$ is not finite dimensional, the white noises are "random distributions" in the following sense: if $\mathcal{H}=L^{2}(X)$, we can take for $\mathcal{H}$ a Sobolev space $H^{s}$ of negative order $s<-\operatorname{dim} X / 2$ and for $A$ the injection from $L^{2}(X)$ into $H^{s}(X)$. So that $w$ can be viewed as a random distribution in $H^{s}(X)$.

\footnotetext{
${ }^{6}$ A Hilbert-Schmidt operator $A$ is an operator whose Schwartz kernel $[A](x, y)$ is in $L^{2}(X \times X)$ and the Hilbert-Schmidt norm $\|A\|_{\mathrm{H}-\mathrm{S}}$ of $A$ is the $L^{2}$ norm of $[A]$.
} 


\subsection{An important example}

Take a self-adjoint operator $\hat{H}$ on $\mathcal{H}$ with compact resolvent. Let us denote by $A_{I}$ the (finite rank) spectral projection onto a compact interval $I$. The random field $f_{I}=A_{I} w$ can also be defined in terms of the spectral decomposition $\left(f_{j}, \lambda_{j}\right)$ of $\hat{H}$. $f_{I}=\sum_{\lambda_{j} \in I} a_{j}(\bar{\omega}) f_{j}$ where the coefficients $a_{j}$ satisfies $\mathbb{E}\left(a_{i}\right)=0$ and $\mathbb{E}\left(a_{i} \overline{a_{j}}\right)=\delta_{i, j}$.

\subsection{Stationary Random fields}

We will now consider time dependent random fields $f: t \rightarrow f(t) \in \mathcal{K}$ with $t \in \mathbb{R}$. Equivalently, our global Hilbert space is now $\mathcal{H}=L^{2}(\mathbb{R}, \mathcal{K})$. We can easily define an action of $\mathbb{R}$ on such a random field. For $T \in \mathbb{R}$, we define $T$. $f$ by $(T$. $f)(t)=f(t-T)$. We will say that

Definition $3.4 f$ is stationary if there exists a group of probability preserving transformations on $\Omega$, denoted by $\left\{X_{T}, T \in \mathbb{R}\right\}$, so that $T .(f(\bar{\omega}))=f\left(X_{T}(\bar{\omega})\right)$.

Let us consider the time dependent fields $f: \mathbb{R} \rightarrow \mathcal{K}$ as elements of a topological vector space $\mathcal{B}$. Stationarity corresponds to the physical intuition that $T$. $f$ is another random choice of the function $f$ (see also Wiener's book [35]).

If $f$ is a stationary random field, the covariance kernel $K\left(t, t^{\prime}\right)=\mathbb{E}(\mid f(t)><$ $\left.f\left(t^{\prime}\right) \mid\right)=K\left(t-t^{\prime}\right)$, where $K(s) \in \operatorname{Herm}(\mathcal{K})$.

Definition 3.5 A stationary random field $f$ is ergodic if any measurable subset of $\Omega$, invariant by the transformations $X_{T}$, is of probability 0 or 1 .

We have then the celebrated

Theorem 3.2 (Birkhoff) If $f$ is stationary and ergodic and $F: \Omega \rightarrow \mathbb{R}$ is integrable with respect to the probability measure, then, for almost every $\bar{\omega} \in \Omega$,

$$
\lim _{T \rightarrow \infty} \frac{1}{T} \int_{0}^{T} F\left(X_{t}(\bar{\omega})\right) d t=\mathbb{E}(F) .
$$

\subsection{Examples of stationary fields}

We will start with a Gaussian white noise $w$ on $L^{2}(\mathbb{R}, \mathcal{K})$. Let us consider a HilbertSchmidt operator $A: L^{2}(\mathbb{R}, \mathcal{K}) \rightarrow \mathcal{K}$. Let us introduce the stationary random field $f$ defined by

$$
f(t)=A(t . w) .
$$

If the Schwartz kernel of $A$ is $a(s, x, y)$ we have, formally,

$$
f(t, x)=\int_{X}|d y| \int_{\mathbb{R}} d s a(s+t, x, y) w(s, y) .
$$

Le us assume that $A w=\int_{\mathbb{R}} \tilde{A}(s) w(s) d s$ with $\tilde{A}(s)$ a Hilbert-Schmidt operator smoothly depending on $s$, with compact support $\subset\left[-t_{0} / 2, t_{0} / 2\right]$. From the formula in Theorem 3.1 the correlation operator is

$$
K(t)=\int_{\mathbb{R}} \tilde{A}(t+s) \tilde{A}(s) d s
$$

which is supported in $\left[-t_{0}, t_{0}\right]$. 
Example 3.2 Stationary scalar noise on the real line: let us take $\mathcal{K}=\mathbb{R}, F \in$ $C_{o}^{\infty}(\mathbb{R}, \mathbb{R}) \sqrt{7}$ and the operator $A$ given by $w \rightarrow \int_{\mathbb{R}} F(-s) w(s) d s$. Then $f$ is the convolution $F \star w$. If we take the Fourier transform w.r.to time, we find for each frequency $\omega \in \mathbb{R} \hat{f}(\omega)=\hat{F}(\omega) \hat{w}(\omega)$, so that $\mathbb{E}\left(|\hat{f}(\omega)|^{2}\right)=|\hat{F}|^{2}(\omega)$. The positive function $\omega \in \mathbb{R} \mapsto|\hat{F}|^{2}(\omega)$ is usually called the power spectrum of the stationary noise $f$.

Example 3.3 Brownian motions: if $\mathcal{K}=\mathbb{R}$ and $w$ is the Gaussian white noise, the Brownian motion is defined by $b(t)=\int_{0}^{t} w(s) d s$, which is in $L^{2}([0, T])$ for all finite $T$. Notice that $b$ is NOT a stationary random field!

\subsection{Random fields from pseudodifferential operators}

The main goal of the present section is to build natural random fields which are non homogeneous with small distances of correlation of the order of $\varepsilon \rightarrow 0$. The noise is non homogeneous in $X$, but could also be non isotropic w.r. to directions.

We will use as Hilbert-Schmidt operators some $\varepsilon$-pseudodifferential operators.

\subsubsection{Random fields from pseudodifferential operators}

Let us take for $\varepsilon$-dependent random fields on a manifold $Z$ (which can be $X \times \mathbb{R}$ ) the image of a white noise on $L^{2}(Z)$ by an $\varepsilon$-pseudodifferential operator $A$ of smooth compactly supported symbol $a(z, \zeta) \in S_{\text {class }}^{-\infty}$ (see Appendix A, Definition 8.1); this operator $A$ is Hilbert-Schmidt.

The correlation $C\left(z, z^{\prime}\right)$ will then be given as the Schwartz kernel of $A A^{\star}$ which is a $\Psi D O$ of principal symbol $|a|^{2}$. We have

$$
C\left(z, z^{\prime}\right)=(2 \pi \varepsilon)^{-d} k\left(z, \frac{z^{\prime}-z}{\varepsilon}\right)+O\left(\varepsilon^{-(d-1)}\right),
$$

where $k(z,$.$) is the Fourier transform with respect to \zeta$ of $|a|^{2}(z, \zeta)$.

This construction gives smooth random fields which can be localised in some very small domains of the manifold $Z$, which are non isotropic (the covariance kernel $C\left(z, z^{\prime}\right)$ for $z^{\prime}$ close to $z$, depends on the direction and not only on the distance $\left.\left|z-z^{\prime}\right|\right)$ and which have small distance of correlations of the order of $\varepsilon$. Moreover it will allow to use techniques of micro-local analysis with the small parameter given by $\varepsilon$.

\subsubsection{Power spectrum and Wigner measures}

The Wigner measures represent the phase space energy densities of a family of functions depending on a small parameter $\varepsilon$.

Definition 3.6 If $f=\left(f_{\varepsilon}\right)$ is a family of functions on $Z$, bounded in $L_{\mathrm{loc}}^{2}(Z)$, the Wigner measures $W_{f}^{\epsilon}$ of $f$ are the measures on the phase space $T^{\star} Z$ defined, for $\varphi \in C_{o}^{\infty}\left(T^{\star} Z\right)$, by (see Appendix A, Definition 8.2)

$$
\int \varphi d W_{f}^{\epsilon}:=\left\langle\mathrm{Op}_{\varepsilon}(\varphi) f_{\varepsilon} \mid f_{\varepsilon}\right\rangle
$$

The measures $d W_{f}^{\varepsilon}$ are the phase space densities of energy of the functions $\left(f_{\varepsilon}\right)$.

We now define:

\footnotetext{
${ }^{7}$ If $X$ is a smooth manifold $C_{o}^{\infty}(X, \mathbb{R})$ denotes the space of smooth functions with compact support
} 
Definition 3.7 The power spectrum of the random field $f=\left(f_{\varepsilon}\right)$ is the phase space measure $\mathcal{P}_{f}^{\varepsilon}$ defined by:

$$
\mathcal{P}_{f}^{\varepsilon}=\mathbb{E}\left(W_{f}^{\varepsilon}\right):
$$

the power spectrum of a random field is the average of its Wigner measures.

Proposition 3.1 The power spectrum $\mathcal{P}$ of $f_{\varepsilon}=\mathrm{Op}(a)$ w, satisfies:

$$
\mathcal{P}_{f}^{\varepsilon}=(2 \pi \varepsilon)^{-d}|a|^{2}(x, \xi)|d x d \xi|+O\left(\varepsilon^{-(d-1)}\right) .
$$

Proof.-

Let us put $A=\mathrm{Op}_{\varepsilon}(a)$, we have

$$
\left\langle\mathrm{Op}_{\varepsilon}(\varphi) A w \mid A w\right\rangle=\left\langle A^{\star} \mathrm{Op}_{\varepsilon}(\varphi) A w \mid w\right\rangle
$$

and $\forall B, \mathbb{E}(\langle B w \mid w\rangle)=\operatorname{trace}(B)$. We get

$$
\mathbb{E}\left(\int \varphi d W_{f}^{\varepsilon}\right)=\operatorname{trace}\left(A^{\star} \mathrm{Op}_{\varepsilon}(\varphi) A\right)
$$

which can be evaluated using the $\Psi \mathrm{DO}$ calculus as

$$
\mathbb{E}\left(\int \varphi d W_{f}^{\varepsilon}\right)=(2 \pi \varepsilon)^{-d} \int \varphi|a|^{2} d x d \xi+O\left(\varepsilon^{-(d-1)}\right) .
$$

\subsubsection{Time dependent random fields}

If $Z=X \times \mathbb{R}$ is the space-time, we will denote by $(x, \xi)$ (resp. $(t, \omega))$ the canonical local coordinates in $T^{\star} X$ (resp. $T^{\star} \mathbb{R}$ ). We will take our random fields as before: $f=L_{\varepsilon} w$; the symbol $l$ of $L_{\varepsilon}=\mathrm{Op}_{\varepsilon}(l)$ is assumed to be $l(x, \xi, \omega) \in S_{\text {class }}^{-\infty}(X \times \mathbb{R})$ (independent of $t$ ). The field $f$ is stationary.

In this case, the correlation is given by:

$$
K(x, y ; t)=\left[L L^{\star}\right](x, y ; 0, t)
$$

which is the Schwartz kernel of a $\Psi D O$ of principal symbol $|l|^{2}(x, \xi ; \omega)$.

Lemma 3.1 The covariance operator $K(t)$ is a $\Psi D O$ in the space $\Psi_{\text {class }}^{-\infty}$ of principal symbol

$$
\frac{1}{2 \pi \varepsilon} \int_{\mathbb{R}}|l|^{2}(x, \xi ; \omega) e^{i t \omega / \varepsilon} d \omega
$$

\section{Correlation for 1-component wave equation}

In this section, we will study the case of a scalar field given as in Section 2.3. First we describe, as a warming up, a case where the correlation is exactly computable, then we compute the asymptotic of the correlation in the "semiclassical case".

\subsection{An example of an exact formula for the correlation}

Let us consider the 1-component "wave equation" (with $\varepsilon=1$ )

$$
\frac{d u}{d t}+k u+i \hat{H}_{0} u=f
$$


where $k>0$ is a constant and $\hat{H}_{0}$ is self-adjoint on $\mathcal{K}$ with a compact resolvent. In particular $\Omega(t)=e^{-k t} U(t)$ where $U(t)$ is the unitary group $U(t)=\exp \left(-i t \hat{H}_{0}\right)$. Let us assume that $\mathbf{f}=\Phi \star A_{I} w$, i.e. $f(t)=A_{I}\left(\int \Phi(t-s) w(s) d s\right)$, with $w$ a Gaussian white noise on $L^{2}(\mathbb{R}, \mathcal{K}), \Phi \in C_{o}^{\infty}(]-t_{0} / 2, t_{0} / 2[; \mathbb{R})$ and $A_{I}=\chi_{I}\left(\hat{H}_{0}\right)$ is the finite rank spectral projection of $\hat{H}_{0}$ associated to the compact interval $I$. The operator $A: w \rightarrow \int_{\mathbb{R}} \Phi(-s) A_{I} w(s) d s$ is clearly Hilbert-Schmidt. From Equation (8), the correlation is given by $K(t)=\Psi(t) A_{I}$ with $\Psi(t)=\int_{\mathbb{R}} \Phi(t+s) \Phi(s) d s$. Using Theorem 1.1, we get

Theorem 4.1 We have the following simple formula for $\tau>t_{0}$ :

$$
C(\tau)=\frac{1}{2 k} F\left(\hat{H}_{0}\right) \Omega(\tau),
$$

with $F(\omega)=\chi_{I}(\omega) \mathcal{F}_{t \rightarrow \omega}\left(\Psi(t) e^{k t}\right)$ (8), which is an exact relation between the correlation and the propagator $P_{0}$ of the wave equation without attenuation.

Remark 4.1 At least formally, we can take $\Phi=\delta(0), I=\mathbb{R}$, then $f=w$ and $F \equiv 1$. The correlation is then equal to the Green's function up to the factor $1 / 2 k$.

Remark 4.2 Assuming that $\hat{H}$ is "real valued" (i.e. $\overline{\hat{H} f}=\hat{H} \bar{f})$, we have $C_{A, B}(-\tau)=$ $\overline{C_{A, B}(\tau)}$.

\subsection{Semiclassical assumptions}

Let us start with the semiclassical Schrödinger like equation

$$
u_{t}+\frac{i}{\varepsilon} \hat{H} u=f
$$

where

- $\hat{H}$ is admissible (Definition 10.1 in Appendix C): $\hat{H}$ is an $\varepsilon$-pseudodifferential operator:

$$
\hat{H}:=\mathrm{Op}_{\varepsilon}\left(H_{0}+\varepsilon H_{1}\right)
$$

with

- The principal symbol $H_{0}(x, \xi): T^{\star} X \rightarrow \mathbb{R}$, which gives the classical ("rays") dynamics, belongs to $\Sigma_{m}$, with $m>0$, and is elliptic of degree $m$ (see Definition 10.11).

- The sub-principal symbol $H_{1}(x, \xi)$ belongs to $\Sigma_{m-1}$ and admits some positivity property which controls the attenuation: there exists $k>0$, such that

$$
h_{1}(x, \xi):=\Im H_{1}(x, \xi) \leq-k, \quad \forall(x, \xi) \in T^{*} X .
$$

- The random field $f$ is given by $f=\operatorname{Op}_{\varepsilon}(l(x, \xi, \omega)) w$ with $w$ a white noise on $X \times \mathbb{R}$ and with $l$ smooth, compactly supported w.r. to $(x, \xi)$ and whose Fourier transform w.r. to $\omega$ is compactly supported in $]-T_{0}, T_{0}[$. The power spectrum of $f$ is $(2 \pi \varepsilon)^{-(d+1)}|l|^{2}(x, \xi, \omega)$. The covariance operator $K(t)$ is a $\Psi$ DO given by Lemma 3.1 and supported in the interval $]-T_{0} \varepsilon, T_{0} \varepsilon[$.

- We introduce some compact energy interval $I$ so that $\operatorname{Support}(l) \subset H_{0}^{-1}(I)$.

Lemma 4.1 Under the previous assumption on $H_{1}$, we can take for $T_{\text {att }}$ (defined in Section 1.1) any number $>1 / k$ provided that $\varepsilon$ is small enough.

\footnotetext{
${ }^{8} \mathcal{F}$ is the Fourier transform $\mathcal{F}(g)(u)=\int \exp (-i u s) g(s) d s$.
} 
Proof.-

$$
\frac{d}{d t}\langle v(t) \mid v(t)\rangle=2 \Re\left\langle v(t) \mid-i \hat{H}_{1}(t) v(t)\right\rangle
$$

and we use Gårding inequality (see [10]): if $a \geq 0, \mathrm{Op}_{\varepsilon}(a) \geq-C$ for any $C>0$ and $\varepsilon$ small enough.

\subsection{Ehrenfest time}

Definition 4.1 (Ehrenfest time) Let $X$ be a smooth compact manifold of dimension d. Let us consider a smooth elliptic Hamiltonian $H_{0}$ on $T^{\star} X$. We fix also some compact energy interval $I$.

- The Liapounov exponent $\left.\Lambda_{I}=\Lambda \in\right] 0,+\infty$ [ is any real number (9) for which the differential of the Hamiltonian flow $\Phi_{t}$ of $H_{0}$ satisfies the following uniform estimate:

$$
\exists C>0, \forall z \in T^{\star} X \text { with } H_{0}(z) \in I, \forall t \geq 0,\left\|d \Phi_{t}(z)\right\| \leq C e^{\Lambda t} .
$$

- Let us denote by $\varepsilon>0$ the semiclassical parameter. The Ehrenfest time, $\left.\left.T_{\text {Ehrenfest }} \in\right] 0,+\infty\right]$ is defined as

$$
T_{\text {Ehrenfest }}:=|\log \varepsilon| / \Lambda .
$$

The intuition behind the definition of the Ehrenfest time is the following one: if $C$ is a phase space cell of diameter $\sim \varepsilon$, the diameter of the cell $\phi_{t}(C)$ remains $\ll 1$ for any time $t$ smaller than $T_{\text {Ehrenfest }}$. We will need a smaller time:

Definition 4.2 Let us give $0<\gamma<\frac{1}{2}$. The time $T_{\gamma}=T_{\gamma}(I)$ is defined as

$$
T_{\gamma}:=\left(\frac{1}{2}-\gamma\right) T_{\text {Ehrenfest }} .
$$

The previous definition will be used via the

Lemma 4.2 Assume that we have a flow $\Phi_{t}$ on a compact manifold $X$ with a Liapounov exponent $\Lambda$, then we have the following uniform estimate

$$
\forall \alpha \in \mathbb{N}^{d}, \forall \Lambda^{\prime}>\Lambda, \quad\left|D^{\alpha} \Phi_{t}(z)\right|=O\left(e^{\Lambda^{\prime}|t||\alpha|}\right) .
$$

\subsection{Main result $(N=1)$}

We get the main result:

Theorem 4.2 With the assumptions and notations of Section 4.2 and $\gamma<\frac{1}{2}$, the correlation is given, for $\tau>0$, by $C(\tau)=\Omega(\tau) \circ \mathrm{Op}_{\varepsilon}(\pi)+R$ where

$$
\begin{aligned}
& \pi(x, \xi)=\int_{0}^{T_{\gamma}(I)} \exp \left(2 \int_{-t}^{0} \Im\left(H_{1}\right)\left(\Phi_{s}(x, \xi)\right) d s\right)|l|^{2}\left(\Phi_{-t}(x, \xi),-H_{0}(x, \xi)\right) d t \\
& \text { belongs to } S_{\frac{1}{2}-\gamma}^{-\infty} \text {, and is supported in } H_{0}^{-1}(I) .
\end{aligned}
$$

\footnotetext{
${ }^{9}$ For technical reasons, not taking the infimum of these $\Lambda$ 's is more convenient
} 
- 1. [No assumption on the attenuation $k]$ we take

$$
\gamma=\gamma_{1}=\frac{k}{2(\Lambda+k)}
$$

and, for any $\alpha<2 \gamma_{1}$, we have the Hilbert-Schmidt estimate

$$
\|R\|_{\mathrm{H}-\mathrm{S}}=O\left(\varepsilon^{\alpha-d / 2}\right)
$$

(the operator $\Omega(\tau) \circ \mathrm{Op}_{\varepsilon}(\pi)$ is Hilbert-Schmidt with an Hilbert-Schmidt norm of the order of $\varepsilon^{-d / 2}$ if $\pi$ is not $\left.\equiv 0\right)$;

2. [Large attenuation] if $k>2 d \Lambda$, we take

$$
\gamma=\gamma_{2}=\frac{k-2 d \Lambda}{2(\Lambda+k)}
$$

and, for any $\beta<2 \gamma_{2}$, for $(A, B, \tau)$ generic (see Definition 9.1), we have the point-wise uniform estimate

$$
[R](A, B)=O\left(\varepsilon^{\beta-\frac{d}{2}}\right) .
$$

Proof.-

From Lemma 8.1, we know that the operator $\mathcal{L}$ introduced in Theorem 1.1 belongs to $\Psi_{\text {class }}^{-\infty}$ and his principal symbol is $|l|^{2}\left(x, \xi,-H_{0}(x, \xi)\right)$. The correlation operator $C(\tau)$ is given (Theorem 1.1) by $C(\tau)=\Omega(\tau) \circ$ $\int_{0}^{\infty} \Omega(u) \mathcal{L} \Omega^{\star}(u) d u$. We split the integral $\int_{0}^{\infty}=\int_{0}^{T_{\gamma}}+\int_{T_{\gamma}}^{\infty}$.

- The first integral is estimated using the long time Egorov Theorem.

- The second one is estimated using the exponential decay of $\Omega(t)$

The main term comes from Theorem 10.2 with the formula for $a_{0}$ given in Theorem 10.1. We split the remainder $R$ into $R=R_{1}+R_{2}$ with

$$
R_{1}=\Omega(\tau) \int_{0}^{T_{\gamma}} r_{1}(s) d s
$$

and $r_{1}(s) \in \varepsilon^{2 \gamma} \Psi_{\frac{1}{2}-\gamma}^{-\infty}$ (using Theorem 10.2)

$$
R_{2}=\int_{T_{\gamma}}^{\infty} \Omega(s+\tau) \mathcal{L} \Omega^{\star}(s) d s
$$

with $\mathcal{L} \in \Psi_{\text {class }}^{-\infty}$.

1. The Hilbert-Schmidt estimate: from Lemma 8.2 we get

$$
\left\|R_{1}\right\|_{\mathrm{H}-\mathrm{S}}=O\left(\varepsilon^{-d / 2} \varepsilon^{2 \gamma}|\log \varepsilon|\right) .
$$

Using the fact that the Hilbert-Schmidt norm of $\mathcal{L}$ is of order $\varepsilon^{-d / 2}$, we have

$$
\left\|R_{2}\right\|_{\mathrm{H}-\mathrm{S}}=O\left(\varepsilon^{-d / 2} \int_{T_{\gamma}}^{\infty} e^{-2 k s} d s\right) .
$$

Using the explicit value $\gamma=\gamma_{1}$, we get the result.

2. The point-wise estimate: 
Lemma 4.3 The Sobolev $H^{s}$ norm of a distribution $u$ in $X$ is, uniformly in $\varepsilon$, equivalent to

$$
\varepsilon^{-s}\left\|\hat{H}^{s / m} u\right\|_{L^{2}}+\|u\|_{L^{2}} .
$$

Taking $s>d$, using Sobolev's embedding Theorem on $X \times X$ and the fact that $\hat{H}$ commutes with $\Omega(s)$, we get the point-wise estimates

$$
\left[R_{2}\right](A, B)=O\left(\varepsilon^{-2 s} \int_{T_{\gamma}}^{\infty}\left\|\Omega(s+\tau) \hat{H}^{s / m} \mathcal{L} \hat{H}^{s / m} \Omega^{\star}(s) d s\right\|_{\mathrm{H}-\mathrm{S}} d s\right)
$$

and

$$
\left[R_{2}\right](A, B)=O\left(\varepsilon^{-2 s} \varepsilon^{(1-2 \gamma) k / \Lambda}\right) .
$$

The result follows from the optimisation $\gamma=\gamma_{2}$.

\subsection{Applications: recovering the Hamiltonian from the Cor- relations}

Theorem 4.3 Let $U$ be the set of $\left(B, \xi_{B}\right) \in T^{\star} X$ so that there exists $t>0$ with $l\left(\Phi_{-t}\left(B, \xi_{B}\right),-H_{0}\left(B, \xi_{B}\right)\right) \neq 0$. For any $\tau_{0}>0$, the restriction of the Hamiltonian $H_{0}$ to $U$ can be recovered from the knowledge of $C(\tau)$ for $0<\tau<\tau_{0}$.

Remark 4.3 The physical meaning of the previous assumption is that the ray $d y$ namics and its Hamiltonian generator $H_{0}$ can be recovered in the domain where the rays cross, in the past time, the support of the power spectrum of the source noise.

Proof.-

Let us assume, for simplicity, that $\partial^{2} H_{0} / \partial \xi^{2}$ is non degenerate. For $\tau>0$ small enough, there is no conjugate points on the unique trajectory from $B$ to $A$ in time $\tau$ contained in $H^{-1}(I)$. From Theorem 4.2 and Appendix B, we get the following expression:

$$
C_{A, B}(\tau)=(2 \pi i \varepsilon)^{-d / 2} c(A, B, \tau) e^{i S(A, B, \tau) / \varepsilon}+R(A, B, \tau)
$$

with

- $c(A, B, \tau)>0$ if $\left(B, \xi_{B}\right) \in U$ (this follows from Section 9.1 and the fact that the principal symbol $\pi$ of $\Pi$ is $>0)$

- The $L^{2}(X \times X)$ norm of $R(., ., \tau)$ is $O\left(\varepsilon^{\alpha-d / 2}\right)$ with $\alpha>0$.

The Theorem follows: $S$ is a generating function for $\Phi_{\tau}$, the flow $\Phi_{\tau}$ determines $H_{0}$ up to a constant. The constant can be extracted from $S$ which is given as the action integral $\int_{\gamma} \xi d x-H_{0} d t$ where $\gamma$ is the trajectory from $B$ to $A$ in time $\tau$.

Without the simplifying assumption, the Theorem still holds: the $\mathrm{H}-\mathrm{S}$ norm of the "Fourier integral operator" $\Omega(\tau) \circ \mathrm{Op}_{\varepsilon}(\pi)$ is of order $\varepsilon^{-d / 2}$. 


\section{The case of multi-component wave equations}

\subsection{Wave equations: source white noise}

Let us take the case of a scalar wave equation with constant damping; the closest results were derived in [33, 21. We will make a formal calculation in the spirit of Remark 4.1. We will consider the wave equation (6), $u_{t t}+2 a u_{t}-\Delta u=f$, with

- $a>0$ is a constant damping coefficient

- $\Delta$ a Riemannian laplacian in some Riemannian manifold $X$, possibly with boundary:

$$
\Delta=g^{i j}(x) \partial_{i j}+b_{i}(x) \partial_{i}
$$

which is self-adjoint with respect to $|d x|$ and appropriate boundary conditions; in fact we could replace the Laplacian by any self-adjoint operator on $X$ !

- $f=f(x, t)$ the source of the noise which will be assumed to be a scalar white noise (homogeneous diffuse field):

$$
\mathbb{E}\left(f(x, s) f\left(y, s^{\prime}\right)\right)=\delta\left(s-s^{\prime}\right) \delta(x-y)
$$

In order to get a readable expression, it is convenient to introduce $Q=\sqrt{-\left(\Delta+a^{2}\right)}$. We get then easily:

$$
u(x, t)=\int_{0}^{\infty} d s \int_{X} e^{-a s}\left[\frac{\sin s Q}{Q}\right](x, y) f(y, t-s)|d y|
$$

where $\sin s Q$ is defined from the spectral decomposition of $Q$ (any choice of the square root gives the same result for $\left.\frac{\sin s Q}{Q}\right)$. We define

$$
G_{a}(t, x, y)=Y(t)\left[e^{-a t} \frac{\sin t Q}{Q}\right](x, y)
$$

with $Y$ the Heaviside function. We will call $G_{a}$ the (causal) Green's function. We can rewrite:

$$
u(x, t)=\int_{\mathbb{R}} d s \int_{X} G_{a}(t-s, x, y) f(y, s)|d y| .
$$

Let us assume now that $f$ is an homogeneous white noise and compute formally the correlation $C_{A, B}(\tau)$.

We get quite easily, using

$$
\begin{gathered}
\sin \alpha \sin \beta=\frac{1}{2}(\cos (\alpha-\beta)-\cos (\alpha+\beta)): \\
C_{A, B}(\tau)=\frac{e^{-a|\tau|}}{4 a}\left[\left(Q^{2}+a^{2}\right)^{-1}\left(\cos \tau Q+\frac{a \sin |\tau| Q}{Q}\right)\right](A, B) .
\end{gathered}
$$

Taking the $\tau$ derivative, we get the simpler formula:

$$
\frac{d}{d \tau} C_{A, B}(\tau)=\left\{\begin{array}{l}
\frac{-1}{4 a} G_{a}(\tau, A, B) \text { for } \tau>0 \\
\frac{1}{4 a} G_{a}(-\tau, A, B) \text { for } \tau<0
\end{array}\right.
$$

This "exact" relation between the correlation and the Green's function in the case of a white noise source field is the starting point of many works in seismology. It has been derived in various ways by several authors in particular R. Weaver and O. Lobkis [18, 31, 33, 21. 
Remark 5.1 The previous calculation can be extended to the more general wave equation (7):

$$
u_{t t}+k u_{t}-\Lambda u=f
$$

with $k>0$ constant, $\Lambda$ self-adjoint and $f$ a white noise. In particular, $C_{A, B}(\tau)$ is an even function of $\tau$. This is the basis of

- Clock synchronisation [22]: if the clocks at the stations $A$ and $B$ are not synchronised, the correlation is even with respect to a shifted origin of times.

- Test of applicability of the theory: in the semiclassical regime studied later, approximate evenness holds. In fact the oscillating part is even because the corrections to the Green's function do not affects the phases. In Theorem 4.2 the principal symbol $\pi$ is $\geq 0$. This is basically due to the time reversibility of the ray dynamics.

\subsection{General wave equations: semiclassics}

\subsubsection{Assumptions}

We want to derive results similar to those of Theorem 4.2 in the case of (multicomponent) wave equations. For that, we will introduce a matrix version of what is done in Section 4 and use the results of Section 8.7. Let us start with the following case of Equation (1):

$$
\frac{\varepsilon}{i} \mathbf{u}_{t}+\hat{H} \mathbf{u}=\frac{\varepsilon}{i} \mathbf{f}
$$

where

- $\hat{H}$ is a matrix of $\varepsilon$-pseudodifferential operators:

$$
\hat{H}:=\mathrm{Op}_{\varepsilon}\left(H_{0}+\varepsilon H_{1}\right)
$$

with (see Appendix A, Section 8.7)

- The principal symbol $H_{0}(x, \xi): T^{\star} X \rightarrow \operatorname{Herm}\left(\mathbb{C}^{N}\right)$ belongs to $\Sigma_{m}^{\mathbb{C}^{N}}, \mathbb{C}^{N}$ and is elliptic of degree $m$ with the eigenvalues $\lambda_{1}(x, \xi)<\cdots<\lambda_{j}(x, \xi)<$ $\cdots<\lambda_{n}(x, \xi)$ of constant multiplicities $m_{j}$; the corresponding eigenspaces $E_{j}(x, \xi) \subset \mathbb{C}^{N}$, of dimension $m_{j}$, are called the polarisations. This assumption is satisfied for the elastic wave equation in a manifold of dimension 3 with $N=6: 2$ eigenvalues $\pm \lambda_{S}$ of multiplicity 2 (the $S$-waves) and 2 eigenvalues $\pm \lambda_{P}$ of multiplicity 1 (the $P$-waves).

- The sub-principal symbol $H_{1}(x, \xi) \in \Sigma_{m}^{\mathbb{C}^{N}}, \mathbb{C}^{N}$ admits some positivity property which controls the attenuation: there exists $k>0$, such that the sub-principal symbols $H_{1, j}, j=1, \cdots, n$, satisfy

$$
\Im H_{1, j} \leq-k<0 \text {. }
$$

- The random field $\mathbf{f}$ is given by $\mathbf{f}=\mathrm{Op}_{\varepsilon}(l(x, \xi, \omega)) \mathbf{w}$ :

- The noises $\mathbf{w}=\left(w_{1}, \cdots, w_{N}\right)$ are independent white noises on $X \times \mathbb{R}$

- The matrix $l$ is smooth, compactly supported w.r. to $(x, \xi)$ and its Fourier transform w.r. to $\omega$ is compactly supported. The power spectrum of $f$ is $(2 \pi \varepsilon)^{-(d+1)} l l^{\star}(x, \xi, \omega)$. 


\subsubsection{The main result}

Using the gauge transform reducing $\hat{H}$ to the block diagonal normal form of Section 8.7.2 we can decompose the correlation into blocks $C_{A, B}^{j, k}(\tau), j, k=1, \cdots, n$.

Theorem 5.1 With the assumptions of Section 5.2.1 the correlations $C_{A, B}^{j, k}(\tau)$ are given

- if $j=k$, as in Theorem 4.0 with $H_{0}=\lambda_{j}$,

- if $j \neq k$ and $l(x, \xi, \omega)$ vanishes for $x$ near $B: C_{A, B}^{j, k}(\tau)=O\left(\varepsilon^{\infty}\right)$.

The proof makes a strong use of the normal form of Section 8.7.2 we use a block decomposition of $\mathcal{L}=\left(\mathcal{L}_{i j}\right)$ in Equation (2) and the block diagonal reduction $\Omega(t)=$ $\left(\Omega_{i}(t)\right)$. We are reduced to the study of

$$
\Omega_{i}(\tau) \int_{0}^{\infty} \Omega_{i}(t) \mathcal{L}_{i j} \Omega_{j}^{\star}(t) d t
$$

If $i=j$, we are in a situation very close to that of a scalar Hamiltonian (Section 4.4), while if $i \neq j$, we can use Lemma 10.2 .

In particular, for the elastic wave equation, if the source noise is supported away from $B$, the correlation $C_{A, B}^{P, S}$ between $\mathrm{S}$-waves and $\mathrm{P}$-waves is very small.

\section{Coda's correlations}

In 5, M. Campillo and A. Paul computed the correlation of coda waves and saw the emergence of the Green's function. Let us try, rather informally, to discuss this observation, even if we do not have mathematical results on it.

Let $u(x, t)$ be the solution of the wave equation with initially localised Cauchy data (delta functions, no randomness in the Cauchy data) at time $t=0$ (an "earthquake") The coda is the field $u(x, t)$ in some window $\left[T_{1}, T_{2}\right]$ with $T_{1}, T_{2}>>0$. Let us look at the correlation

$$
C_{A, B}(\tau)=\lim _{T_{1}, T_{2} \rightarrow \infty} \frac{1}{T_{2}-T_{1}} \int_{T_{1}}^{T_{2}} u(A, t+\tau) \bar{u}(B, t) d t
$$

assumed to exist. Introducing the propagator $P$, we get:

$$
C_{A, B}(\tau)=\int_{X}|d x| P(\tau, A, x)\left(\lim _{T_{1}, T_{2} \rightarrow \infty} \frac{1}{T_{2}-T_{1}} \int_{T_{1}}^{T_{2}} u(x, t) \bar{u}(B, t) d t\right) .
$$

So that the question is reduced to the study of the limits of $\frac{1}{T_{2}-T_{1}} \int_{T_{1}}^{T_{2}} u(A, t) \bar{u}(B, t) d t$ as $T_{1}, T_{2}, T_{2}-T_{1} \rightarrow \infty$. If we have some nice (smooth?) limit $K(A, B)$, then $C_{A, B}(\tau)=[\Omega(\tau) \circ \hat{K}](A, B)$.

Looking at some semiclassical limit, this is closely related to the following result by R. Schubert 26]: if the classical dynamics is hyperbolic and the time $t$ of the order of the Ehrenfest time, the smoothed Wigner measures ("Husimi" measures) of $u(t,$.$) are equidistributed on each energy shell. Does this uniformity of the energy$ distribution extend to the wave functions themselves, in particular to the phases?

\section{Using surface waves in order to image the inner crust}

In real applications to seismology, the fields are recorded at the surface of the Earth. Moreover the main contribution to the correlations comes from the surface waves: 
- They have a smaller time decay than the body waves.

- The source of the noise itself (interaction with atmosphere and ocean) is located at the surface.

The Earth's crust acts as a wave guide. This implies that we really have to look at a $2 D$ version of the wave equation: the wave equation for surface wave is an effective wave equation on the surface. This equation is given in terms of eigenvalues of a vertical SturmLiouville equation. Solving an inverse spectral problem for these Sturm-Liouville equation allows (in principle!) to image the crust. The inverse spectral problem is solved in [7].

In this Section, we will discuss these effective Hamiltonians in the scalar model of the acoustic wave equation and show how one can reduce the problem of imaging the Earth's crust to an inverse spectral problem.

\subsection{A mathematical model}

We work locally in $X=\left\{(\mathbf{x}, z) \in \mathbb{R}^{2} \times \mathbb{R} \mid z \leq 0\right\}$ so that $z=0$ is the surface. We will consider the very simple case of an acoustic wave equation near the origin of $X$. Let us give a function

$$
N(\mathbf{x}, Z): \mathbb{R}^{2} \times \mathbb{R}_{-} \rightarrow \mathbb{R}_{+}
$$

which satisfies the following assumptions:

1. There exists $N_{\infty}>0$ and $Z_{0}<0$, so that $N(\mathbf{x}, Z)=N_{\infty}$ for $Z \leq Z_{0}$.

2. For every $\mathbf{x} \in \mathbb{R}^{2}, \inf _{Z} N(\mathbf{x}, Z)=N_{0}(\mathbf{x})>0$ and $N_{0}(\mathbf{x})<N_{\infty}$ for $\mathbf{x}$ close to 0

3. $N$ is $C^{1}$ w.r. to $Z$ and the map $\mathbf{x} \rightarrow N(\mathbf{x},$.$) , from \mathbb{R}^{2}$ into the Banach space $C^{1}\left(\left[Z_{0}, 0\right], \mathbb{R}\right)$, is smooth.

Let us introduce a small parameter $\varepsilon>0$ and the following acoustic wave equation where

$$
\begin{gathered}
n(\mathbf{x}, z)=N\left(\mathbf{x}, \frac{z}{\varepsilon}\right): \\
\left\{\begin{array}{l}
u_{t t}-\operatorname{div}(n \operatorname{grad} u)=0 \\
\frac{\partial u}{\partial z}(\mathbf{x}, 0)=0
\end{array}\right.
\end{gathered}
$$

We plan to see that Equation (15) admits, as $\varepsilon \rightarrow 0$, asymptotic solutions of frequency of order $\varepsilon^{-1}$ located near the surface. Moreover these solutions are determined by solving an effective pseudodifferential equation on the surface $\partial X=$ $\mathbb{R}^{2} \times\{0\}$. In order to show that we can apply Theorem 8.3 of Appendix A, we will first introduce the operator $H_{0}$ in Section 7.3. It will be a Sturm-Liouville operator in the variable $Z$.

\subsection{Discussion of the assumptions on the model}

The scalar acoustic wave equation (15) is a simplified model of the elastic wave equation. We could work with the elastic wave equation, but it will be more complicated and the main ideas would be the same.

The function $n$ represent the square of the local propagation speed (group velocity). The formula (14) means that the speed admits fast variations in the vertical directions and is smooth in the horizontal direction. It is a plausible assumption that the the geological structure near the surface of the earth is "stratified".

Assumption (1) on $N$ means that the speed is constant far from the surface. It is an assumption which could be removed at the price of a technical work. 
Assumption (2) implies that the speed is somewhere smaller than the speed at large depth. This kind of assumption is usually satisfied in seismology where the speed of elastic waves into sediments layers is smaller than the speed inside the rocks below the sediments.

Assumption (3) means that we need less regularity in the vertical direction. It could be relaxed using piecewise smoothness w.r. to $Z$, but at the price of a rather technical work: the domain of the Sturm-Liouville operator introduced below would then depend on $\mathbf{x}$.

\subsection{A family of Sturm-Liouville operators}

Let us consider, for each $(\mathbf{x}, \xi) \in T^{\star} \partial X$, the self-adjoint differential operator $L_{\mathbf{x}, \xi}$ on the half line $Z \leq 0$, with Neumann boundary condition at $Z=0$, defined by:

$$
L_{\mathbf{x}, \xi} v:=-\frac{d}{d Z}\left(N(\mathbf{x}, Z) \frac{d v}{d Z}\right)+N(\mathbf{x}, Z)|\xi|^{2} v .
$$

The domain $D \subset L^{2}\left(\mathbb{R}^{-}\right)$of $L_{\mathbf{x}, \xi}$ is

$$
D:=H^{2}\left(\mathbb{R}^{-}\right) \cap\left\{v \mid v^{\prime}(0)=0\right\}
$$

which is independent of $(\mathbf{x}, \xi)$.

The spectrum of $L_{\mathbf{x}, \xi}$ consists of a finite discrete spectrum and a continuous spectrum $\left[N_{\infty}|\xi|^{2},+\infty\left[\right.\right.$. $L_{\mathbf{x}, \xi}$ admits, for $\xi$ large enough, a non empty discrete spectrum of simple eigenvalues

$$
N_{0}(\mathbf{x})|\xi|^{2}<\lambda_{1}(\mathbf{x}, \xi)<\cdots<\lambda_{j}(\mathbf{x}, \xi)<\cdots<\lambda_{k}(\mathbf{x}, \xi)<N_{\infty}|\xi|^{2},
$$

which depend smoothly of $(\mathbf{x}, \xi)$. In order to see that, we can interpret $\mathcal{L}_{\hbar}=$ $|\xi|^{-2} L_{\mathbf{x}, \xi}$ as a semiclassical Schrödinger type operator with an effective Planck constant $\hbar=|\xi|^{-1}$ and a principal symbol

$$
p_{\mathbf{x}}(Z, \zeta)=N(\mathbf{x}, Z)\left(\zeta^{2}+1\right)
$$

which admits a "well" because of Assumption 2 in Section 7.1 on $N$.

We should however take care of the fact that the number $k$ of eigenvalues depends on $(\mathbf{x}, \xi)$ and goes to $\infty$ as $\xi$ does. This will give birth to mode conversions between surface waves and body waves. To my knowledge, such mode conversions have not yet been studied from a mathematical point of view.

\subsection{Wave guides as an adiabatic limit}

We will rewrite Equation (15) as an operator valued pseudodifferential equation as in Appendix A, Section 17.4] in the spirit of the book [29], Chapter 3 on "spaceadiabatic perturbation theory".

Putting

$$
u(t, \mathbf{x}, z)=v\left(t, \mathbf{x}, \frac{z}{\varepsilon}\right)
$$

we get the following wave equation for $v$ :

$$
\varepsilon^{2} v_{t t}-\left(\partial_{z}\left(N \partial_{z} v\right)+\varepsilon^{2} N \Delta_{\mathbf{x}} v+\varepsilon^{2} \operatorname{grad}_{\mathbf{x}} N \cdot \operatorname{grad}_{\mathbf{x}} v\right)=0,
$$

which can be rewritten, using Weyl quantisation in the variable $\mathbf{x}$ :

$$
\varepsilon^{2} v_{t t}+\mathrm{Op}_{\varepsilon}\left(L_{\mathbf{x}, \xi}-i \varepsilon \xi \cdot \operatorname{grad}_{\mathbf{x}} N\right) v=0 .
$$

Putting $H_{0}((\mathbf{x}, \xi))=L_{\mathbf{x}, \xi}$ and $H_{1}\left((\mathbf{x}, \xi)=-i \xi \cdot \operatorname{grad}_{\mathbf{x}} N\right.$, we will apply Theorem 8.3 in Appendix A. Let us choose, for $(\mathbf{x}, \xi)$ close to 0 , some eigenvalue $\lambda_{j}(x, \xi)$ of 
$L_{\mathbf{x}, \xi}$ with a normalised eigenfunction $\varphi_{j}(\mathbf{x}, \xi, Z)$. Then we have the existence of a propagation mode of the form

$$
v(t, \mathbf{x}, z)=w(t, \mathbf{x})\left(\varphi_{j}(\mathbf{x}, \xi, z / \varepsilon)+\varepsilon \cdots\right)
$$

where $w$ satisfying the scalar wave equation:

$$
\varepsilon^{2} w_{t t}+\mathrm{Op}_{\varepsilon}\left(\lambda_{j}(x, \xi)+\cdots\right) w=0 .
$$

For each eigenvalue $\lambda_{j}$, we can then apply the results of Section 5 .

From the passive imaging method, we can hope to recover the effective Hamiltonians $\pm \sqrt{\lambda_{j}}$. Solving an inverse spectral problem for the Sturm-Liouville operator $L_{\mathbf{x}, \xi}$ allows in principle to recover the function $n(\mathbf{x}, z)$ which encodes the physics of the propagation medium.

From the paper [7, we know that the function $N$ can be recovered from the large $\xi$ asymptotic of the $\lambda_{j}$ 's.

\section{Appendix A: a review about pseudodifferential operators}

We review the basic definitions and properties of pseudodifferential operators ( $\Psi$ DO's). Pseudodifferential operators (first called singular integral operators by Calderón and Zygmund) where developed in order to get a class of operators generalising linear differential operators and containing approximate inverses of elliptic operators. They became a basic tool in the theoretical study of linear differential operators with variable coefficients. A more general theory was then developed including a small parameter, here denoted by $\left.\varepsilon \in] 0, \varepsilon_{0}\right]$. Several textbooks are now available [6, [10, [11, [30].

\subsection{Basic calculus}

We will define the pseudodifferential operators ( $\Psi$ DO's) on $\mathbb{R}^{d}$. $\Psi$ DO's on manifolds are defined locally by the same formulae.

Definition 8.1 (Classical symbols) - The space $\Sigma_{k}$ of symbols of degree $k$ is the space of smooth functions $p: T^{\star} \mathbb{R}^{d} \rightarrow \mathbb{C}$ which satisfy

$$
\forall \alpha, \beta \in \mathbb{N}^{d},\left|D_{x}^{\alpha} D_{\xi}^{\beta} p(x, \xi)\right| \leq C_{\alpha, \beta}\langle\xi\rangle^{k},
$$

with $\langle\xi\rangle=1+\|\xi\|$.

- A classical symbol of degree $m$ is a family of functions

$$
p_{\varepsilon}: T^{\star} \mathbb{R}^{d} \rightarrow \mathbb{C}
$$

depending of some small parameter $\varepsilon>0$ which admits, as $\varepsilon \rightarrow 0$, an asymptotic expansion

$$
p_{\varepsilon} \equiv \sum_{j=0}^{\infty} \varepsilon^{j} p_{j}(x, \xi)
$$

with $p_{j} \in \Sigma_{m}$. We will denote this space by $S_{\text {class }}^{m}$.

Definition 8.2 (Pseudodifferential operators) A classical $\varepsilon$-pseudodifferential operator $P_{\varepsilon}(a \Psi D O)$ of degree $m$ on $\mathbb{R}^{d}$ is given by the (distribution) kernel

$$
\left[P_{\varepsilon}\right]\left(x, x^{\prime}\right)=(2 \pi \varepsilon)^{-d} \int_{\mathbb{R}^{d}} e^{i\left\langle x-x^{\prime} \mid \xi\right\rangle / \varepsilon} p_{\varepsilon}\left(\frac{x+x^{\prime}}{2}, \xi\right)|d \xi|
$$


where $p_{\varepsilon}(x, \xi)$, the so-called (total) symbol of $P$, is in $S_{\text {class }}^{m}$.

We will denote $P_{\varepsilon}=\mathrm{Op}_{\varepsilon}\left(p_{\varepsilon}\right)$ and by $\Psi_{\text {class }}^{m}$ the space of classical $\Psi$ DO's of degree $m$. The operator $P_{\varepsilon}$ is called the Weyl-quantisation of $p_{\varepsilon}$.

The operators $P_{\varepsilon}$ act from $C_{o}^{\infty}$ into $C^{\infty}$. The kernel of $P_{\varepsilon}$ is given by:

$$
\left[P_{\varepsilon}\right]\left(x, x^{\prime}\right)=(2 \pi \varepsilon)^{-d} \tilde{p_{\varepsilon}}\left(\frac{x+x^{\prime}}{2}, \frac{x^{\prime}-x}{\varepsilon}\right)
$$

with $\tilde{p}_{\varepsilon}$ the partial Fourier transform of $p_{\varepsilon}(x, \xi)$ w.r. to $\xi$. Very often, one is only able to compute the symbol $p_{0}$ which is called the principal symbol of $P$. The term $p_{1}$ is called the sub-principal symbol.

The most basic fact about $\Psi D O$ 's is the fact they form a (non-commutative) algebra: if $P_{\varepsilon}=\mathrm{Op}_{\varepsilon}\left(p_{\varepsilon}\right)$ and $Q_{\varepsilon}=\mathrm{Op}_{\varepsilon}\left(q_{\varepsilon}\right)$, we have $P_{\varepsilon} Q_{\varepsilon}=\mathrm{Op}_{\varepsilon}\left(p_{\varepsilon} q_{\varepsilon}+O(\varepsilon)\right)$. The composition formula for the total symbol is called the Moyal $\star$-product $\mathrm{Op}_{\varepsilon}(a) \circ$ $\mathrm{Op}(b)=\mathrm{Op}_{\varepsilon}(a \star b)$ and is given by

$$
a \star b \equiv a b+\frac{\varepsilon}{2 i}\{a, b\}+\sum_{j=2}^{\infty} \varepsilon^{j} P_{j}(a, b)
$$

where

- $\{a, b\}$ is the Poisson bracket

- $P_{j}(a, b)$ is a bi-linear bi-differential operator, homogeneous of degree $j$ with respect to $a$ and $b$.

\subsection{The symbols $S_{\delta}^{m}$}

In order to formulate the Egorov Theorem for long times, we will need more sophisticated classes of symbols.

Definition $8.3\left(S_{\delta}^{m}\right)$ For any real number $m$ and any $\delta$ with $0 \leq \delta<\frac{1}{2}$, a symbol $a \in S_{\delta}^{m}$ is a smooth function on $T^{\star} X$ depending on $\varepsilon$ which satisfies

$$
\forall \alpha \in \mathbb{N}^{2 d}, \exists C_{\alpha}>0, \forall(x, \xi) \in T^{\star} X, \quad\left|D_{x, \xi}^{\alpha} a(x, \xi)\right| \leq C_{\alpha} \varepsilon^{-\delta|\alpha|}\langle\xi\rangle^{m} .
$$

We have $S_{\text {class }}^{m} \subset S_{0}^{m}$.

We can associate to $a \in S_{\delta}^{m}$ a pseudodifferential operator $\operatorname{Op}_{\varepsilon}(a) \in \Psi_{\delta}^{m}$ using formula (17). Such operators obey a nice calculus, see [10, 14]: if, for $j=1,2$, $a_{j} \in S_{\delta_{j}}^{m_{j}}, A_{j}:=\operatorname{Op}_{\varepsilon}\left(a_{j}\right)$ and $\delta:=\max \left(\delta_{1}, \delta_{2}\right)$, we have $A_{1} \circ A_{2}=\operatorname{Op}_{\varepsilon}\left(a_{1} \star a_{2}\right)$ with $a_{1} \star a_{2} \in S_{\delta}^{m_{1}+m_{2}}$ and admits the asymptotic expansion given by the Moyal $\star$-product. We have $P_{j}\left(a_{1}, a_{2}\right) \in \varepsilon^{-j\left(\delta_{1}+\delta_{2}\right)} S_{\delta}^{m_{1}+m_{2}}$. In particular $\left[A_{1}, A_{2}\right]=$ $\frac{\varepsilon}{i} \mathrm{Op}_{\varepsilon}\left(\left\{a_{1}, a_{2}\right\}\right)+\mathrm{Op}_{\varepsilon}(b)$ with $b \in \varepsilon^{2\left(1-\delta_{1}-\delta_{2}\right)} S_{\delta}^{m_{1}+m_{2}}$.

Theorem 8.1 (Calderón-Vaillancourt) The operators of $\Psi_{\delta}^{0}$ extend to operators from $L^{2}$ to $L^{2}$, uniformly continuous in $\varepsilon$. The operators in $\Psi_{\delta}^{m}$ extends to operators from $L^{2}$ into the Sobolev space $H^{-m}$ with a norm of order $O\left(\varepsilon^{m}\right)$.

\subsection{Asymptotic expansions}

The basic tool in order to find a $\Psi D O$ is an inductive construction based on the realisation of any asymptotic expansion: given a sequence $a_{j} \in S_{\delta}^{m}$ and $l_{1}=0<$ $l_{2}<\cdots<l_{j}<\cdots$ and $l_{j} \rightarrow+\infty$ as $j \rightarrow \infty$, there exists $a \in S_{\delta}^{m}$ such that

$$
a \equiv \sum_{j=1}^{\infty} \varepsilon^{l_{j}} a_{j}
$$


meaning that

$$
\forall n, a-\sum_{j=1}^{n-1} \varepsilon^{l_{j}} a_{j} \in \varepsilon^{l_{n}} S_{\delta}^{m} .
$$

Such an $a$ is uniquely defined modulo a remainder term $r$ which lies in $\varepsilon^{\infty} S_{\delta}^{m}$. The corresponding $\Psi$ DO $\mathrm{Op}_{\varepsilon}(a)$ is well defined modulo $O\left(\varepsilon^{\infty}\right)$. This implies, if $m \leq 0$ and $a$ compactly supported in $x$, that the $L^{2} \rightarrow L^{2}$ norm of $\mathrm{Op}_{\varepsilon}(r)$ is $O\left(\varepsilon^{\infty}\right)$.

\subsection{Microlocalisation}

A family $\left(u_{\varepsilon}\right)_{\varepsilon \rightarrow 0}$ of functions on $X$ is said to be admissible if the $L^{2}$ norm on any compact set is at most of polynomial growth with respect to $\varepsilon^{-1}$.

The family of functions $\left(u_{\varepsilon}\right)$ is said to be $O\left(\varepsilon^{\infty}\right)$ near a point $(x, \xi) \in T^{\star} X$ if $\exists \varphi \in$ $C_{o}^{\infty}(X), \varphi\left(x_{0}\right) \neq 0$ and the $\varepsilon$-Fourier transform $\mathcal{F}_{\varepsilon}\left(\varphi u_{\varepsilon}\right)(\xi):=(2 \pi \varepsilon)^{-d / 2} \int e^{-i\langle x \mid \xi\rangle / \varepsilon} u_{\varepsilon}(x) d x$ satisfies $\mathcal{F}_{\varepsilon}\left(\varphi u_{\varepsilon}\right)(\xi)=O\left(\varepsilon^{\infty}\right)$ uniformly for $\xi$ close to $\xi_{0}$.

The micro-support $\operatorname{MS}\left(u_{\varepsilon}\right)$ of $u_{\varepsilon}$ is the set of all $(x, \xi)$ 's at which $u_{\varepsilon}$ is not $O\left(\varepsilon^{\infty}\right)$. For any $\Psi \mathrm{DO} A$, we have $\operatorname{MS}\left(A u_{\varepsilon}\right) \subset \operatorname{MS}\left(u_{\varepsilon}\right)$.

If $U$ is an open subset of $T^{\star} X$ and if $u_{\varepsilon}$ and $v_{\varepsilon}$ are admissible, we will shortly denote $u_{\varepsilon}=v_{\varepsilon}+O\left(\varepsilon^{\infty}\right)$ in $U$ if $\operatorname{MS}(u-v) \cap U=\emptyset$.

\subsection{Functional calculus and and an useful Lemma}

A simple case of the functional calculus for IDO's is as follows:

Theorem 8.2 Let $f \in C_{o}^{\infty}(\mathbb{R})$ and $A=\mathrm{Op}_{\varepsilon}\left(a_{0}+\varepsilon a_{1}+\cdots\right) \in \Psi_{\text {class }}^{m}$ with $a_{0}$ elliptic (see Section 10.1) and real. Then one can define $f(A)$ using the spectral theory and $f(A) \in \Psi_{\text {class }}^{-\infty}$ with principal symbol $f\left(a_{0}\right)$.

Remark 8.1 The previous Theorem is usually stated for A self-adjoint (see [28] Chap. 8). It can be extended to the case where the sub-principal symbol is not real, with a very close proof.

Lemma 8.1 Let us consider the operator $\mathcal{L}$, given by

$$
\mathcal{L}:=\frac{1}{2 \pi \varepsilon} \iint \Omega(-t) e^{i t \omega / \varepsilon} \operatorname{Op}_{\varepsilon}^{x}(L(x, \xi, \omega)) d \omega d t
$$

with $L \in S_{\text {class }}^{-\infty}(X \times \mathbb{R})$ with $L(x, \xi, \omega)$ having an $\omega$-Fourier transform compactly supported as a function of $(x, \xi, t)$. Here $\Omega(t)=\exp (-i t \hat{H} / \varepsilon)$ where $\hat{H}$ satisfies the assumptions of Section 4.2. Then $\mathcal{L}$ belongs to $\Psi_{\text {class }}^{-\infty}$ and its principal symbol is given by $l_{0}(x, \xi)=L\left(x, \xi,-H_{0}(x, \xi)\right)$.

The integral giving $\mathcal{L}$ is supported w.r. to $t$ in the interval $\left[-t_{0}, t_{0}\right]$. Using a change of variable $t=\varepsilon t^{\prime}$ in the Definition of $\mathcal{L}$, the proof is a simple corollary of the functional calculus of $\Psi$ DO's: if $A=\mathrm{Op}_{\varepsilon}(a)$ with $a$ compactly supported, the operator $B=\exp \left(i t^{\prime} \hat{H}\right) A$ belongs to $\Psi_{\text {class }}^{-\infty}$ and the principal symbol of $B$ is $b=\exp \left(i t^{\prime} H_{0}\right) a$; because the function $\exp (i t \cdot)$ is not compactly supported, we need first to rewrite $A=F(\hat{H}) A$ with $F \in C_{o}^{\infty}$ and then use the functional calculus with the function $f=F \exp (i t \cdot)$.

\subsection{Hilbert-Schmidt estimate}

Lemma 8.2 If $P \in \Psi_{\delta}^{-\infty}(X)$ with $X$ a compact manifold, $P$ is Hilbert-Schmidt with $\|P\|_{\mathrm{H}-\mathrm{S}}=O\left(\varepsilon^{-d / 2}\right)$.

Proof.- 
$P^{\star} P \in \Psi_{\delta}^{-\infty}$ is trace class (it has a smooth kernel) and its trace is easily seen to be $O\left(\varepsilon^{-d}\right)$.

\subsection{Multicomponent operators}

\subsubsection{Systems of $\Psi \mathrm{DO}$}

Definition 8.4 Let $E$ and $F$ be 2 Banach spaces $\Sigma_{m}^{E, F}$ is the space of symbols of degree $m$ with values in the Banach space $\mathcal{L}(E, F)$ of continuous linear maps from $E$ into $F$.

Similarly, we can define the spaces $S_{\text {class }}^{m, E, F}, \Psi_{\text {class }}^{m, E, F}, \cdots$. The operators of $\Psi_{\text {class }}^{m, E, F}$ send functions on $X$ with values in $E$ into functions on $X$ with values in $F$.

Definition 8.5 $H_{0} \in \Sigma_{m}^{E, F}$ is elliptic if $H_{0}(x, \xi)$ is invertible for large $\xi$ 's and the inverse is in $\Sigma_{-m}^{F, E}$.

Matrix valued $\Psi$ DO's have properties similar to scalar $\Psi$ DO's.

\subsubsection{Reduction}

Let $\mathcal{H}$ be an Hilbert space and $D \subset \mathcal{H}$ a dense sub-space.

Definition 8.6 $A$ matrix valued Hamiltonian $H_{0}: T^{\star} X \rightarrow \operatorname{Herm}(\mathcal{H})$ is an element of $\Sigma_{m}^{D, \mathcal{H}}$ : for every $(x, \xi) \in T^{\star} X, H_{0}(x, \xi)$ is a self-adjoint operator with domain $D \subset \mathcal{H}$.

Let us consider a classical symbol $H_{\varepsilon} \equiv H_{0}+\varepsilon H_{1}+\cdots \in S_{\text {class }}^{m, D, \mathcal{H}}$ with $H_{0}$ a matrix values Hamiltonian. Let us consider near some point $z_{0} \in T^{\star} X$ an eigenvalue $\lambda_{0}(z)$ of constant multiplicity $k$ and eigenspace $E(z)$ (the polarisation space) and assume that $\lambda_{0}(z)$ is an isolated point in the spectrum of $H_{0}(z)$.

Then we have, inspired from [13, 29]:

Theorem 8.3 In some neighbourhood of $z_{0}$, there exists

- $A \mathcal{L}(\mathcal{H})$-valued $\Psi D O \hat{U}=\mathrm{Op}_{\varepsilon}\left(U_{0}+\varepsilon U_{1}+\cdots\right)$ with $U_{0}(z)$ unitary.

- A classical symbol in $S_{\text {class }}^{m, \mathbb{C}^{k}, \mathbb{C}^{k}} \lambda(z)=\lambda_{0}(z) \operatorname{Id}+\varepsilon \lambda_{1}(z)+\cdots$

- $A$ symbol $K$ with values in $\mathcal{L}(\mathcal{D} \cap F, F)$ with $F=E^{\perp}$ with $\lambda_{0}(z) \notin \operatorname{Spectrum}\left(K_{0}(z)\right)$,

so that

$$
U^{-1} \mathrm{Op}_{\varepsilon}(H) U \equiv\left(\begin{array}{cc}
\mathrm{Op}_{\varepsilon}(\lambda) & 0 \\
0 & \mathrm{Op}_{\varepsilon}(K)
\end{array}\right)
$$

near $z_{0}$.

Moreover $\lambda_{1}(z)=\pi H_{1} \pi+R_{1}$ with $\pi$ the orthogonal projector onto $E$ and $R_{1}$ self-adjoint.

The proof is done by induction on the powers of $\varepsilon$ : at the first step, we choose an unitary gauge transform $U(z) \in \mathcal{L}(\mathcal{H})$ so that

$$
U^{-1} H_{0} U=\left(\begin{array}{cc}
\lambda_{0} \mathrm{Id} & 0 \\
0 & K_{0}
\end{array}\right)
$$

with $\lambda_{0} \mathrm{Id}-K_{0}$ invertible. At the next step, we try to find

$$
B=\left(\begin{array}{ll}
0 & b \\
c & 0
\end{array}\right)
$$


so that

$$
(1-\varepsilon B) \star\left(\left(\begin{array}{cc}
\lambda_{0} \mathrm{Id} & 0 \\
0 & K_{0}
\end{array}\right)+\varepsilon \tilde{H}_{1}\right) \star(1+\varepsilon B)=\left(\begin{array}{cc}
\lambda_{0} \mathrm{Id}+\varepsilon \lambda_{1} & 0 \\
0 & K_{0}+\varepsilon K_{1}
\end{array}\right)+O\left(\varepsilon^{2}\right) .
$$

Collecting terms in $\varepsilon^{1}$, we get the following equations:

$$
b\left(\lambda_{0} \mathrm{Id}-K_{0}\right)=\beta,\left(\lambda_{0} \mathrm{Id}-K_{0}\right) c=-\gamma
$$

with

$$
\tilde{H}_{1}=\left(\begin{array}{cc}
\alpha & \beta \\
\gamma & \delta
\end{array}\right)
$$

These equations can be solved with the assumption that $\lambda_{0} \operatorname{Id}-K_{0}$ is invertible.

Remark 8.2 In the Theorem 8.3, we do not need that $\mathrm{Op}_{\varepsilon}(H)$ is self-adjoint (only $\mathrm{Op}_{\varepsilon}\left(H_{0}\right)$ is self-adjoint), neither that the symbol of $\mathrm{Op}_{\varepsilon}(H)$ takes values in bounded operators on $\mathcal{H}$.

Remark 8.3 The Theorem 8.3 will be used as well to reduce a multi-component wave equation to scalar equation in Section 5.2 and as tool for adiabatic limits in Section 7.4

Corollary 8.1 Assuming that all eigenvalues $\lambda_{1}<\cdots<\lambda_{j}<\cdots<\lambda_{n}$ of $H_{0}(x, \xi)$ are of constant multiplicities $m_{j}, j=1, \cdots, n$, the Hamiltonian $\hat{H}$ admits a microlocal normal form with diagonal blocks of sizes $m_{j} \times m_{j}$ and principal symbols of the $j$-th block $\lambda_{j} \operatorname{Id}_{\mathbb{C}^{m_{j}}}$.

The sub-principal symbol of the $j$-th block is $H_{1, j}:=\pi_{j} H_{1} \pi_{j}+R_{j}$ where $R_{j}$ is self-adjoint while $\pi_{j}$ is the orthogonal projection on $E_{j}$; it means that, as quadratic forms, $H_{1, j}$ is the restriction of $H_{1}$ to $E_{j}$.

The formula for the sub-principal symbols is a direct consequence of [13].

\subsubsection{Wave equations}

Let us consider the wave equation (7)

$$
u_{t t}+A u_{t}-\Lambda u=0
$$

with $\varepsilon^{2} \Lambda=\mathrm{Op}_{\varepsilon}\left(H_{0}\right)\left(H_{0}\right.$ elliptic) and assume w.l.o.g. that $-\Lambda$ is $>0$ so that $\Lambda_{\frac{1}{2}}=\varepsilon \sqrt{-\Lambda}$ is also a $\Psi$ DO. Putting

$$
U:=\left(\begin{array}{c}
\Lambda_{\frac{1}{2}} u \\
-i \varepsilon u_{t}
\end{array}\right)
$$

Equation (7) reads as:

$$
\frac{\varepsilon}{i} U_{t}+\left(\begin{array}{cc}
O & -\Lambda_{\frac{1}{2}} \\
-\Lambda_{\frac{1}{2}} & -i \varepsilon A
\end{array}\right) U=0
$$

which is of the form

$$
\frac{\varepsilon}{i} U_{t}+\hat{H} U=0
$$

with $\hat{H}$ an elliptic $\Psi$ DO. 


\subsubsection{Extension of Lemma 8.1}

Let us consider $a \in \mathcal{S}\left(\mathbb{R}_{\omega}, L\left(\mathbb{C}^{N}\right)\right)$ and $H_{0}$ an $N \times N$ Hermitian matrix, we define $a\left(H_{0}\right)$ as

$$
a\left(H_{0}\right):=\frac{1}{2 \pi} \int e^{i t H_{0}} \mathcal{F} a(t) d t .
$$

The following Lemma is proved the same way as Lemma 8.1 .

Lemma 8.3 Let us consider the operator $\mathcal{L}$, given by

$$
\mathcal{L}:=\frac{1}{2 \pi \varepsilon} \iint \Omega(-t) e^{i t \omega / \varepsilon} \operatorname{Op}_{\varepsilon}^{x}(L(x, \xi, \omega)) d \omega d t
$$

with $L \in S_{\text {class }}^{-\infty, \mathbb{C}^{N}, \mathbb{C}^{N}}(X \times \mathbb{R})$ with $L(x, \xi, \omega)$ having an $\omega$ Fourier transform compactly supported as a function of $(x, \xi, t)$. Here $\Omega(t)=\exp (-i t \hat{H} / \varepsilon)$ where $\hat{H}$ satisfies the assumptions of Section 5.2. Then $\mathcal{L}$ belongs to $\Psi_{0}^{-\infty, \mathbb{C}^{N}, \mathbb{C}^{N}}$ and his principal symbol is given by $l_{0}(x, \xi)=L\left(x, \xi,-H_{0}(x, \xi)\right)$.

\section{Appendix B: asymptotics of the Green's func- tion and classical dynamics}

\subsection{The scalar case}

We want to describe quite explicitly the propagator $P(t, x, y)=[\Omega(t)](x, y)$ with $\Omega(t)=\exp (-i t \hat{H} / \varepsilon), \hat{H}$ as in 4.2 . We will do that in the energy interval $I$; it means that we will only describe the action of $\Omega(t)$ on functions whose micro-supports are in $H_{0}^{-1}(I)$.

To the Hamiltonian $H_{0}: T^{\star} X \rightarrow \mathbb{R}$, we associate the ray dynamics defined by

$$
\frac{d x_{j}}{d t}=\frac{\partial H_{0}}{\partial \xi_{j}}, \frac{d \xi_{j}}{d t}=-\frac{\partial H_{0}}{\partial x_{j}} .
$$

Let us denote by $\phi_{t}$ the flow of the Hamiltonian vector field $X_{H_{0}}$ defined in Equation (18). The main result is that $P(t, . .$.$) is a Fourier Integral Operator$ associated to the flow $\phi_{t}$. For simplicity, we will only describe the so-called generic case where $P$ admits a WKB expansion.

If $x_{0}, y_{0} \in X$ and $\phi_{t}\left(y_{0}, \eta_{0}\right)=\left(x_{0}, \xi_{0}\right)$, we say that $x_{0}$ and $y_{0}$ are not conjugate along the ray $\gamma(s)=\phi_{s}\left(y_{0}, \eta_{0}\right), 0 \leq s \leq t$, if the differential $\delta \eta \rightarrow d \phi_{t}(0, \delta \eta)$ is invertible at the point $\left(y_{0}, \eta_{0}\right)$. This implies that for $(x, y)$ close enough to $\left(x_{0}, y_{0}\right)$ there exists an unique $(y, \eta)$ close to $\left(y_{0}, \eta_{0}\right)$ with $\phi_{t}(y, \eta)=(x, \xi)$. In this case the action $S_{\gamma}(t, x, y)$ is defined by $S_{\gamma}(t, x, y)=\int_{\gamma} \xi d x-H_{0}(x, \xi) d t . S_{\gamma}(t, . .$.$) is a$ generating function of $\phi_{t}$ near $\left(y_{0}, \eta_{0}\right)$, meaning that $\phi_{t}\left(y,-\partial S_{\gamma} / \partial y\right)=\left(x, \partial S_{\gamma} / \partial x\right)$ for $(y, \eta)$ close to $\left(y_{0}, \eta_{0}\right)$.

The action $S_{\gamma}$ is useful in order to describe a WKB expansion of the propagator $P(t, x, y)$ for $(x, y)$ close to $\left(x_{0}, y_{0}\right): P(t, x, y)$ is a sum of contributions $P_{\gamma}(t, x, y)$ of rays going from $y$ to $x$ in the time $t$.

Assuming that $x$ and $y$ are not conjugate along $\gamma$, we have

$$
P_{\gamma}(t, x, y) \equiv(2 \pi \varepsilon)^{-d / 2}\left(\sum_{j=0}^{\infty} \varepsilon^{j} a_{j, \gamma}(t, x, y)\right) e^{i S(t, x, y) / \varepsilon} .
$$

Moreover $a_{0, \gamma}(t, x, y)>0$ if $t$ is small enough. 
Definition 9.1 We will say that $(x, y, t)$ is generic if $x$ and $y$ are not conjugate along any $\gamma$ so that $\gamma(0)=x, \gamma(t)=y$ and $\gamma \subset H_{0}^{-1}(I)$.

If $(x, y, t)$ is generic, there is only a finite number of rays from $y$ to $x$ in time $t$ included in $H_{0}^{-1}(I)$.

Dispersive rays: we say that the dynamics generated by $H_{0}$ is dispersive if $\left(H_{0}\right)_{\xi \xi}$ is invertible. This means that the map $\xi \mapsto v(\xi):=\partial H_{0} / \partial \xi$ ( $v$ is the speed) is a local diffeomorphism. If this is the case, $(t, x, y)$ is generic for $x$ close enough to $y$.

The dispersion relation: the principal symbol of the wave equation is $\omega+$ $H_{0}(x, \xi): T^{\star}(X \times \mathbb{R}) \rightarrow \mathbb{R}$. It is clear enough that the ray dynamics is determined by the hypersurface $\mathbb{D}:=\left\{(x, \xi, \omega) \mid \omega+H_{0}(x, \xi)=0\right\}$. This surface is called the dispersion relation. This terminology does not mean that $H_{0}$ is dispersive!

\subsection{The matrix case}

Let us assume that $H_{0}$ is matrix valued as in Section 5.2. The dispersion relation is then the hypersurface

$$
\operatorname{det}\left(\omega \mathrm{Id}-H_{0}(x, \xi)\right)=\mathbb{D}(x, \xi, \omega)=0,
$$

we can (at least outside a mode conversion set), rewrite

$$
\mathbb{D}(x, \xi, \omega)=\Pi_{j=0}^{M}\left(\omega-\lambda_{j}(x, \xi)\right)^{d_{j}}
$$

which gives $M$ ray dynamics $\lambda_{j}$ associated to different polarisations

$$
E_{j}=\operatorname{ker}\left(H_{0}(x, \xi)-\lambda_{j}(x, \xi) \mathrm{Id}\right) .
$$

\section{Appendix C: long time Egorov Theorem}

The main reference for this part is 3 where the authors get only an operator norm estimates for the remainder. The proofs of reference [3] can be extended in order

- to get a point-wise result under some ellipticity assumption

- to cover the case of manifolds

- to use a local Liapounov exponent: the Liapounov exponent in some energy interval $I$

- to allow an attenuation term.

\subsection{Egorov theorem}

Definition 10.1 An admissible Hamiltonian of degree $m>0$ is $\hat{H}:=\mathrm{Op}_{\varepsilon}\left(H_{0}+\right.$ $\left.\varepsilon H_{1}\right)$ with

- $H_{0} \in \Sigma_{m}$ a real valued function which is elliptic:

$$
\exists c, C>0, \forall(x, \xi) \in T^{\star} X, \quad H_{0}(x, \xi) \geq C\langle\xi\rangle^{m}-c .
$$

- $H_{1} \in \Sigma_{m-1}$ and $h_{1}=\Im H_{1} \leq-k \leq 0$ everywhere in $T^{\star} X$.

Example 10.1 A typical admissible Hamiltonian is the damped Schrödinger operator $\hat{H}=-\varepsilon^{2} \Delta+V(x)-i \varepsilon a(x)$ with $V(x) \geq 0$ and $a(x) \geq 0$. 
We define the dynamics, for $t \geq 0$, by:

$$
\Omega(t)=\exp (-i t \hat{H} / \varepsilon) .
$$

Theorem 10.1 (Egorov Theorem) Let $\hat{H}=\mathrm{Op}_{\varepsilon}(H)$ be an admissible Hamiltonian and $A(0)=\operatorname{Op}_{\varepsilon}(a)$ with $a \in C_{o}^{\infty}\left(H_{0}^{-1}(I)\right)$ with $I$ a compact interval. Then the operator $A(t)=\Omega(t) A(0) \Omega^{\star}(t)$ is, for $t$ fixed, a pseudodifferential operator whose symbol $a(t) \equiv \sum_{j=0}^{\infty} \varepsilon^{j} a_{j}(t)$ belongs to $S_{\text {class }}^{-\infty}$ and satisfies

$$
a_{0}(t)(z)=\exp \left(2 \int_{-t}^{0} h_{1}\left(\Phi_{s}(z)\right) d s\right) a\left(\Phi_{-t}(z)\right) .
$$

Let us remark that the previous result is a priory uniform only in a fixed interval $t \in\left[0, T_{0}\right]$ independent of $\varepsilon$.

Let us sketch the proof. $A(t)$ is the value for $s=t$ of the solution $B(s)$ of the ODE

$$
\frac{d}{d s} \Omega(t-s) B(s) \Omega^{\star}(t-s)=0, B(0)=A(0) .
$$

We have

$$
\frac{d}{d s} \Omega(t-s) B(s) \Omega^{\star}(t-s)=\Omega(t-s)\left(B^{\prime}(s)+\frac{i}{\varepsilon}\left(\hat{H} B(s)-B(s) \hat{H}^{\star}\right)\right) \Omega^{\star}(t-s) .
$$

Trying $B(s)=\mathrm{Op}_{\varepsilon}\left(b_{0}(s)+\varepsilon b_{1}(s)+\cdots\right)$ and using the Moyal product, we have to solve

$$
b^{\prime}(s)+\frac{i}{\varepsilon}\left(\left(H_{0}+\varepsilon H_{1}\right) \star b(s)-b(s) \star\left(H_{0}+\varepsilon H_{1}\right)\right) \equiv 0 .
$$

We get the following equations by looking at the $\varepsilon^{j}$ term:

$$
\frac{d}{d t} b_{j}+\left\{H_{0}, b_{j}\right\}_{1}-2 h_{1} b_{j}=c_{j},
$$

with $c_{0}=0$ and, for $j \geq 1, c_{j}=\sum_{k=0}^{j-1} P_{k, j}\left(b_{k}\right)$ where the $P_{k, j}$ 's are linear differential operators constructed by using the Moyal product with $H_{0}$ and $H_{1}$. If $\tilde{B}(s)=$ $\mathrm{Op}_{\varepsilon}\left(b_{0}(s)+\varepsilon b_{1}(s)+\cdots\right)$, we have

$$
\frac{d}{d s} \Omega(t-s) \tilde{B}(s) \Omega^{\star}(t-s)=\Omega(t-s) R(s) \Omega^{\star}(t-s)
$$

with $R(s) \in \varepsilon^{\infty} \Psi_{\text {class }}^{-\infty}$.

It remains to prove that $\Omega(t-s) R(s) \Omega^{\star}(t-s)$ is $O\left(\varepsilon^{\infty}\right)$ as an operator from the Sobolev space $H^{-s}$ into the space $H^{s}$, for any $s$. From the Calderón-Vaillancourt Theorem 8.1, it is enough to prove that $L^{2} \rightarrow L^{2}$ norm of $\hat{H}^{s / m} \Omega(t-s) R(s) \Omega^{\star}(t-$ $s)\left(\hat{H}^{\star}\right)^{s / m}$ is $O\left(\varepsilon^{\infty}\right)$. This is clear because the powers of $\hat{H}$ (resp. of $\left.\hat{H}^{\star}\right)$ commute with $\Omega(t-s)$ (resp. $\left.\Omega^{\star}(t-s)\right)$.

\subsection{Long time Egorov Theorem}

We want to extend Egorov Theorem to times going to infinity when $\varepsilon \rightarrow 0$. From Lemma 4.2, we get (see [3]):

Lemma 10.1 Let $a_{0} \in C_{o}^{\infty}\left(T^{\star} X\right)$ and $I=H_{0}\left(\operatorname{Support}\left(a_{0}\right)\right)$. Let $T_{\gamma}(I)$ be the time defined in Definition 4.2. Then, the function $a_{0}(t)$ belongs to $S_{\delta}^{-\infty}$ with $\delta=$ $\frac{1}{2}-\gamma<\frac{1}{2}$ for all times $t \in\left[0, T_{\gamma}(I)\right]$, and the constants in the estimates of the derivatives are uniform in $t$. Similarly, the symbolds of higher order $\varepsilon^{j} a_{j}$ belong to $\varepsilon^{j(1-2 \delta)} S_{\delta}^{-\infty}$. 
The main result is:

Theorem 10.2 (Long time Egorov Theorem) Let $\hat{H}=\mathrm{Op}_{\varepsilon}(H)$ be an admissible Hamiltonian (Section 10.1) and $a \in C_{o}^{\infty}\left(T^{\star} X\right)$. For any $t \in\left[0, T_{\gamma}\right]$ and with uniform symbol estimates w.r. to $t$, the operator $A(t):=\Omega(t) A \Omega^{\star}(t)$ is a pseudodifferential operator, the total symbol of which is given as in the classical Egorov Theorem 10.1 by

$$
A(t) \equiv \mathrm{Op}_{\varepsilon}\left(\sum_{j=0}^{\infty} \varepsilon^{j} a_{j}(t)\right),
$$

where $\varepsilon^{j} a_{j}(t) \in \varepsilon^{j(1-2 \delta)} S_{\delta}^{-\infty}$.

More precisely, for any $M$ and $k$, there exists $N$ so that if $R_{N}(t)=A(t)-$ $\mathrm{Op}_{\varepsilon}\left(\sum_{j=0}^{N} \varepsilon^{j} a_{j}(t)\right)$, the $C^{k}$ norm of the kernel of $R_{N}(t)$ satisfies, uniformly for $t \in\left[0, T_{\gamma}\right]$, the bound

$$
\left\|\left[R_{N}(t)\right]\right\|_{C^{k}(X \times X)}=O\left(\varepsilon^{M}\right) .
$$

Remark 10.1 The long time Egorov Theorem was first proved by Bambusi, Graffi and Paul [1] and improved by Bouzouina and Robert [3].

Our estimate of the remainder term is better than the one given in [3] which is only an $L^{2}$ operator norm. We need the ellipticity of $H_{0}$.

Using Lemma 10.1, the proof is the same as for a fixed time interval (see Section 10.1).

\subsection{Integrals with two dynamics}

Lemma 10.2 Let us assume that we have two admissible Hamiltonians $\hat{H}_{ \pm}=$ $\mathrm{Op}_{\varepsilon}\left(H_{0, \pm}+\varepsilon H_{1, \pm}\right)$ with $k_{ \pm}>0$. Let us define the operator

$$
J:=\int_{0}^{\infty} \Omega_{+}(t+\tau) \mathrm{Op}_{\varepsilon}(a) \Omega_{-}^{\star}(t) d t,
$$

with $a \in C_{o}^{\infty}\left(T^{\star} X\right)$. If we assume that, for $z \in \operatorname{Support}(a), H_{0,+}(z) \neq H_{0,-}(z)$ (no mode conversions occur in the support of a), then

$$
J=\varepsilon \Omega_{+}(\tau) K+R
$$

with $K$ a $\Psi D O$ of principal symbol $k_{0}=-i a /\left(H_{0,+}-H_{0,-}\right)$ and $R \in \varepsilon^{\infty} \Psi^{-\infty}$.

In particular, if a vanishes in some neighbourhood of $B,[J](A, B)=0\left(\varepsilon^{\infty}\right)$.

Proof.-

There exists some $\Psi$ DO $K$ so that $\mathrm{Op}_{\varepsilon}(a)=-i\left(\hat{H}_{+} K-K \hat{H}_{-}\right)^{\star}+r$ with $r \in \varepsilon^{\infty} \Psi^{-\infty}$. $J$ can be rewritten as

$$
J=\varepsilon \int_{0}^{\infty} \frac{d}{d t}\left(\Omega_{+}(t+\tau) K \Omega_{-}^{\star}(t)\right) d t+\int_{0}^{\infty}\left(\Omega_{+}(t+\tau) r \Omega_{-}^{\star}(t)\right) d t .
$$

Integration by part gives the result. 


\section{Appendix D: stationary Gaussian fields are er- godic}

This section is inspired from [2]. Let us define

$$
C_{T}(\tau, A, B):=\frac{1}{T} \int_{0}^{T} u(A, t) \otimes \overline{u(B, t-\tau)} d t,
$$

where $u$ is the causal solution of Equation(11). We then have

Theorem 11.1 Let us assume that $f(., t)=K w(., t)$ where $w(x, t)$ is a (Gaussian) white noise on $X \times \mathbb{R}$ and $K: \mathcal{H} \rightarrow \mathcal{H}$. Then

1. If $K$ is Hilbert-Schmidt, $C_{T}(\tau, A, B) \rightarrow \mathbb{E}(C(\tau, A, B))$, as $T \rightarrow \infty$, almost surely for almost every pair $(A, B)$.

2. If $K$ is smoothing and $\hat{H}$ elliptic, $C_{T}(\tau, A, B) \rightarrow \mathbb{E}(C(\tau, A, B))$, as $T \rightarrow \infty$, almost surely in the $C^{\infty}(X \times X)$ topology.

Proof.-

1. We will assume w.l.o.g. that $u$ is scalar valued and real and use the following classical identity: if $f=\left(f_{1}, f_{2}, f_{3}, f_{4}\right): \Omega \rightarrow \mathbb{R}^{4}$ is a Gaussian random variable,

$$
\mathbb{E}\left(f_{1} f_{2} f_{3} f_{4}\right)=\mathbb{E}\left(f_{1} f_{2}\right) \mathbb{E}\left(f_{3} f_{4}\right)+\mathbb{E}\left(f_{1} f_{3}\right) \mathbb{E}\left(f_{2} f_{4}\right)+\mathbb{E}\left(f_{1} f_{4}\right) \mathbb{E}\left(f_{2} f_{3}\right) .
$$

We have

$\mathbb{E}\left(\left\|C_{T}(\tau)\right\|_{\mathrm{H}-\mathrm{S}}^{2}\right)=\frac{1}{T^{2}} \int_{0}^{T} d t \int_{0}^{T} d t^{\prime} \int_{X \times X} d A d B \mathbb{E}\left(u(A, t) u(B, t-\tau) u\left(A, t^{\prime}\right) u\left(B, t^{\prime}-\tau\right)\right)$.

We apply the identity (20) and get:

$\mathbb{E}\left(\left\|C_{T}(\tau)\right\|_{\mathrm{H}-\mathrm{S}}^{2}\right)=\left\|\mathbb{E}\left(C_{T}(\tau)\right)\right\|_{\mathrm{H}-\mathrm{S}}^{2}+\frac{1}{T^{2}} \int_{0}^{T} d t \int_{0}^{T} d t^{\prime} \int_{X \times X} d A d B(I I+I I I)$,

with

$$
I I=\mathbb{E}\left(u(A, t) u\left(A, t^{\prime}\right)\right) \mathbb{E}\left(u(B, t-\tau) u\left(B, t^{\prime}-\tau\right)\right)
$$

and

$$
I I I=\mathbb{E}\left(u(A, t) u\left(B, t^{\prime}-\tau\right)\right) \mathbb{E}\left(u(A, t-\tau) u\left(B, t^{\prime}\right)\right) .
$$

For example, we have

$\mathbb{E}\left(u(A, t) u\left(B, t^{\prime}-\tau\right)\right)=\left[\int_{0}^{\infty} d s \int_{0}^{\infty} d s^{\prime} \Omega(s) K K^{\star} \Omega^{\star}\left(s^{\prime}\right) \delta\left(t-s=t^{\prime}-s^{\prime}-\tau\right)\right](A, B)$

whose $L^{2}$ norm as a function of $(A, B)$ is estimated as $O\left(\exp \left(-\mid t-t^{\prime}+\right.\right.$ $\tau \mid))$. Using Cauchy-Schwarz inequality and the fact that a product of two Hilbert-Schmidt operators is trace class, we get

$$
\left|\frac{1}{T^{2}} \int_{0}^{T} d t \int_{0}^{T} d t^{\prime} \int_{X \times X} d A d B(I I+I I I)\right|=O(1 / T) .
$$

Standards argument involving Tchebichev inequality allow to conclude.

2. The second assertion is proved in a similar way using the Sobolev embeddings, like at the end of the proof of Theorem 10.1 . 


\section{References}

[1] D. Bambusi, S. Graffi \& T. Paul. Long time semiclassical approximation of quantum flows: A proof of Ehrenfest time. Asymptotic Analysis 21:149-160 (1999).

[2] C. Bardos, J. Garnier \& G. Papanicolaou. Identification of Green's Functions Singularities. Inverse Problems. 24:015011 (2008).

[3] A. Bouzouina \& D. Robert. Uniform semiclassical estimates for the propagation of quantum observables. Duke Math. Jour. 111(2):223-252 (2002).

[4] F. Brenguier, N. M. Shapiro, M. Campillo, V. Ferrazzini, Z. Duputel, O. Coutant \& A. Nercessian. Towards Forecasting Volcanic Eruptions using Seismic Noise. Nature Geoscience doi:10.1038/ngeo104 (2008).

[5] M. Campillo \& A. Paul. Long range correlations of seismic codas. Science 299:547-549 (2003).

[6] Y. Colin de Verdière. Méthodes semi-classiques et théorie spectrale. Lecture Notes in Preparation http://www-fourier.ujf-grenoble.fr/ ycolver/

[7] Y. Colin de Verdière. A semiclassical inverse problem II: reconstruction of the potential. ArXiv: math-ph/08021643.

[8] A. Derode, E. Larose, M. Tanter, J. de Rosny, A. Tourin, M. Campillo \& M. Fink. Recovering the Green's function from field-field correlations in an open scattering medium. J. Acoust. Soc. Am. 113:2973-2976 (2004).

[9] A. Derode, E. Larose, M. Campillo \& M. Fink. How to estimate the Green's function of a heterogeneous medium between to passive sensors? Application to acoustic waves. Applied Physics Lett. 83(15):3054-3056 (2003).

[10] M. Dimassi \& J. Sjöstrand. Spectral Asymptotics in The Semi-Classical Limit. London Math. Soc. Lecture Notes Ser. 268 (1999).

[11] J. Duistermaat. Fourier Integral Operators. Birkhäuser, Boston, 1996.

[12] T. Duvall, S. Jefferies, J. Harvey \& M. Pomerantz. Time-distance in helioseismology. Nature 362:430-432 (1993).

[13] C. Emmrich \& A. Weinstein. Geometry of the Transport Equation in Multicomponent WKB Approximation. Commun. Math. Phys. 176:701-711 (1996).

[14] L. Evans \& M. Zworski. Lectures on semiclassical analysis (Version 0.2). Available at http://math.berkeley.edu/ zworski/

[15] I.M. Gel'fand \& N.Y. Vilenkin. Generalised functions, vol. 4: applications to harmonic analysis. Academic Press, 1964.

[16] P. Gouédard, L. Stéhly, F. Brenguier, M. Campillo, Y. Colin de Verdière, E. Larose, L. Margerin, P. Roux, F.J. Sánchez-Sesma, N. Shapiro \& R. Weaver. Cross-correlation of random fields: mathematical approach and applications. Geophysical prospecting 56:375-393 (2008).

[17] L. Hörmander. The spectral function of an elliptic operator. Acta Mathematica 12:193-218 (1968).

[18] O.I. Lobkis \& R.L. Weaver. On the emergence of Green's function in the correlations of a diffuse field. J. Acoust. Soc. Am. 110:3011-3017 (2001). 
[19] J. Rauch \& M. Taylor. Decay of Solutions of Non-dissipative Hyperbolic Systems on Compact Manifolds. Comm. Pure Appl. Math. 28:501-523 (1975).

[20] M. Reed \& B. Simon, Methods of modern Math. Phys. III.

[21] Ph. Roux, K. Sabra \& W. Kupperman. Ambient noise cross correlation in free space: Theoretical approach. J. Acoust. Soc. Am. 117:79-84 (2005).

[22] K. Sabra, P. Roux, A. Thode, G. D'Spain, W. Hogliss \& W. Kuperman. Using Ocean Ambient Noise for Array Self-Localization and Self-Synchronization. IEEE J. of Oceanic engineering 30:338-346 (2005).

[23] F. Sanchez-Sesma, J. Pérez-Ruiz, M. Campillo \& F. Luzón. The elastodynamic 2D Green's function retrieval from cross-correlation: the canonical inclusion problem. Geophysical Research letters 33:13305 (2006).

[24] N. Shapiro \& M. Campillo. Emergence of broadband Rayleigh waves from correlations of the ambient seismic noise. Geophys. Res. Lett. 31:L07614 (2004).

[25] N. Shapiro, M. Campillo, L. Stehly \& M. Ritzwoller. High Resolution Surface Wave Tomography From Ambient Seismic Noise. Science 307:1615 (2005).

[26] R. Schubert. Semiclassical behaviour of expectation values in time evolved Lagrangian states for large times. Commun. Math. Phys. 256:239:254 (2005).

[27] L. Schwartz. Radon measures on arbitrary topological spaces and cylindrical measures. Oxford University Press, 1973.

[28] J. Sjöstrand. Projecteurs adiabatiques du point de vue pseudo-différentiel. $C$. R. Acad. Sci. Paris, Série 1, 317:217-220 (1993).

[29] S. Teufel. Adiabatic perturbation theory in quantum dynamics. Lecture notes in mathematics 1821 (2003).

[30] F. Trèves. Introduction to pseudo differential and Fourier integral operators. Plenum Press, New York, 1980.

[31] R.L. Weaver \& O.I. Lobkis. On the emergence of Green's function in the correlations of a diffuse field: pulse echo using thermal phonon's. Ultrasonics 40:435-439 (2002).

[32] R.L. Weaver \& O.I. Lobkis. Ultrasonics without a source: thermal fluctuation correlations at MHz frequencies. Phys. Rev. Lett. 87(13):134301-134304 (2001).

[33] R.L. Weaver \& O.I. Lobkis. Diffuse fields in open systems and the emergence of the Green's functions (L). J. Acoust. Soc. Am. 116(5):1-4 (2004).

[34] R.L. Weaver. Information from seismic noise. Science 307:1568-1569 (2005).

[35] N. Wiener. Extrapolation, Interpolation and Smoothing of Stationary Time Series. MIT and John Wiley, 1950.

[36] K. Yosida. Functional Analysis. Springer-Verlag, Grundlheren der Math. Wiss. 123, 1965. 